\title{
Approche globale de besoins non satisfaits en planification familiale au Mali.
}

\author{
Rwenge Mburano ${ }^{1} \&$ Kanambaye Saidou ${ }^{2}$
}

\author{
${ }^{1}$ Institut de Formation et de Recherche Démographiques (IFORD), Yaoundé, Cameroun \\ ${ }^{2}$ Population Services International (PSI), Bamako, Mali \\ Pour les correspondances: rwenge_mburano@yahoo.fr
}

\section{Résumé}

Contexte : Le Mali est l'un des pays d'Afrique de l'Ouest où la fécondité est élevée et stable et la prévalence de la contraception moderne est faible. A cet effet, la présente étude s'est fixée comme objectif de chercher les facteurs associés aux Besoins Non Satisfaits (BNS) en Planification Familiale (PF) dans ce pays.

Données et méthodes : Les données utilisées sont celles des EDS qui y ont été réalisées en 199596,2006 et 2012-13. Leurs analyses ont été effectuées en recourant aux tableaux croisés et statistiques du chi-deux et aux modèles multivariés de régression logistique.

Résultats : En 1995-96 la proportion d'enfants décédés, le nombre d'enfants survivants et les attitudes des couples vis-à-vis de la PF contribuent le plus à l'explication de la variation du risque de BNS en PF pour espacer les naissances; en 2006 et 2012-13, on retrouve en plus les indicateurs de l'offre des services de PF. Quant aux BNS pour limiter les naissances, quelle que soit la période, le nombre d'enfants survivants explique seul presque la totalité de sa variation. Concernant le total des BNS en PF, ses facteurs explicatifs les plus importants sont similaires à ceux du risque de BNS en PF pour espacer les naissances. Les femmes les plus concernées au Mali par les BNS en PF ont un nombre élevé d'enfants survivants, ont moins expérimenté les décès d'enfants et ne sont pas informées sur la PF dans les centres de santé pendant leurs consultations médicales.

Conclusion : L'amélioration de la qualité des services de PF et de la disponibilité des contraceptifs de longue durée d'action, et l'intégration santé infantile et PF devraient être renforcées au Mali pour y réduire la prévalence des BNS en PF.

Mots clés: Facteurs associés, Approche globale, Besoins Non Satisfaits, Planification Familiale, Mali, Afrique subsaharienne.

\section{Abstract}

Background: Mali is one of the West African countries where fertility is high and stable and the prevalence of modern contraceptive is low. Therefore, this study aimed at searching factors associated with the unmet-need for Family Planning (FP) among married women in Mali.

Data source and method: The data used are those of DHS carried out in this country in 1995-96, 2006 and 2012-13. Their analyses were carried out using cross-tabulations and chi-square statistics and multivariate logistic regression models.

Results: In 1995-96, the proportion of dead children, the number of surviving children and the attitudes of couples vis-à-vis FP contribute the most to the explanation of the variance of the unmet-need for birth-spacing; in 2006 and 2012-13, the indicators of the supply of FP services are also associated with this dependent variable. Concerning the unmet-need for birth-limiting, whatever the period, the number of surviving children explains itself its variance. Regarding the total of the unmet-need, its most important factors are similar to those of the unmet-need for birth-spacing. Women who are more concerned in Mali by the unmet-need for FP have a high number of surviving children, have experienced less child deaths and are not informed about FP in health centers during their medical consultations.

Conclusion: Improving the supply of FP services, in terms of their quality and the availability of longacting contraceptives, and the integration of child health and FP should therefore be strengthened in Mali to significantly reduce in this country the prevalence of the unmet-need for FP.

Keywords: Associated factors, Global Approach, Unmet-Need, Family Planning, Mali, Sub-Saharan Africa. 


\section{Introduction}

L'utilisation de la contraception influence la santé reproductive et participe à l'amélioration des conditions de vie des familles (Gribble et Voss, 2009 ; WHO, 2009 ; WHO, 20II). Par ailleurs, au niveau macro, les pays où la fécondité est plus faible et la croissance démographique plus lente connaissent un taux de productivité plus élevé, font davantage d'économies et investissent de manière plus efficace (UNFPA et Path, 2008). A cela s'ajoute le fait que la Planification Familiale (PF) est une pratique déterminante pour l'atteinte du dividende démographique: une fécondité jugulée aboutit notamment à une population dans laquelle les individus en âge de travailler sont plus nombreux que ceux à leur charge, à savoir les enfants et les individus âgés.

Le Mali, comme d'autres pays africains, a déjà pris conscience des conséquences néfastes d'une forte fécondité et mis en place une politique de population, dans laquelle s'inscrivent ses programmes de PF, mais force est de constater que la prévalence contraceptive chez les femmes en union est encore très faible dans ce pays (7\% en 1996, $8 \%$ en 200 l et 2006 et $10 \%$ en 20I2-20I3 (Traoré et Ballo, 2002; Dembele et Keita, 2007 ; CPS/SSDSPF, INSTAT/MPATP, INFO-STAT et ICF International, 20|4). Cependant, la disparité est grande à l'intérieur de ce pays, particulièrement entre le milieu urbain et le milieu rural : la prévalence contraceptive chez les femmes en union résidant en milieu rural est de $3 \%$ en $1996,5 \%$ en 200 l et 2006 et $6,8 \%$ en $2012-13$ tandis que chez les citadines elle est respectivement de $16 \%, 18 \%, 15 \%$ et $21,8 \%$ (Traoré et Ballo, 2002; Dembele et Keita, 2007 ; CPS/SSDSPF, INSTAT/MPATP, INFO-STAT et ICF International, 20I4).

Le problème de faible prévalence des méthodes contraceptives se pose donc avec acuité au Mali et une des solutions efficaces à ce problème serait de réduire le niveau des Besoins Non Satisfaits (BNS) en PF, qui était en 2006 dans ce pays de $31,3 \%$ et en 20I2-13 de $26 \%$ (Dembele et Keita, 2007; CPS/SSDSPF, INSTAT/MPATP, INFO-STAT et ICF International, 20I4). D'où la pertinence des recherches qui s'intéressent à la caractérisation des individus ou des couples qui sont plus concernés que d'autres au Mali par ce problème et à la recherche des facteurs associés. Ceci est d'autant plus important que les BNS s'inscrivent en violation du droit fondamental des femmes et des hommes de contrôler leur propre fécondité et de choisir le nombre d'enfants qu'ils veulent et l'espacement qu'ils désirent entre leurs naissances.
On retrouve dans la littérature existante plusieurs études sur les BNS en PF mais une seule, notamment celle de Mariko et al. (2009), porte sur le Mali. Les critiques ci-après peuvent lui être adressées. i) Comme les données à partir desquelles les facteurs de ce phénomène social y ont été mis en évidence sont anciennes, les recommandations programmatiques auxquelles elle est parvenue ne sont plus d'actualité ; en effet, les changements qui ont eu lieu au Mali de 2006 à 20I 2-13 peuvent s'être répercutés sur les facteurs du phénomène étudié. ii) La recherche des facteurs des BNS en PF n'y a pas été précédée par une réflexion théorique. Son apport dans l'amélioration des connaissances sur le sujet estdonc limité. Par ailleurs, bon nombre d'études réalisées dans d'autres contextes africains ont privilégié une seule approche explicative du phénomène étudié, soit l'approche offre (Belohlav et Nolan, 2013 ; Arends-Kuenning et Kessy, 2007), soit l'approche demande (Kourouma, 20I I), et pourtant pour bien connaître les facteurs du phénomène étudié au Mali, il serait pertinent de s'orienter vers une approche globale. En effet, dans le contexte africain, la disponibilité des services de PF n'implique pas nécessairement leur utilisation par la population, un autre facteur important est l'existence de la demande de la PF dans la population. De même, lorsque celle-ci est faite dans un contexte où l'offre est quantitativement et/ou qualitativement faible, le niveau des BNS dans ce domaine serait important dans la population.

Les questions auxquelles cette étude essaye d'apporter des éléments de réponse sont alors les suivantes: (i) La qualité des services de PF est-elle un facteur important des BNS en PF au Mali? (ii) Qu'en est-il des caractéristiques propres à la femme et de ses rapports avec son conjoint? En d'autres termes, ses caractéristiques socioculturelles, socioéconomiques et sociodémographiques jouentelles un rôle important dans l'ensemble des facteurs? Qu'en est-il des rapports de genre au sein du couple? Pour y arriver, l'étude est organisée en cinq parties: (i) Approche théorique; (ii) Méthodologie; Résultats; (iv) Conclusion et recommandations.

\section{Approche théorique}

L'examen des études antérieures a révélé qu'il existe plusieurs approches explicatives des BNS en PF dans le contexte africain qui peuvent être en deux groupes: approche offre des services et approche demande. 


\section{Approche offre des services de PF}

Selon cette approche, la prévalence des BNS en PF dépend des éléments des cadres légal et juridique relatifs à la PF, des politiques et programmes de PF mis en place et de la disponibilité, l'accessibilité et la qualité des services de PF.

Akoto et Kamdem (200I) suggèrent qu'en Afrique subsaharienne la disponibilité et l'accessibilité des services de PF et leur qualité sont intimement liées à des choix politiques, eux-mêmes dépendant des moyens des Etats et de leur position sur les questions de population. Le cadre institutionnel mis en place peut donc ne pas faciliter l'atteinte des objectifs de certains programmes dans le domaine de la PF, du fait de son incohérence avec la réalité sociale du milieu. Par ailleurs, le décalage fréquent entre les discours des décideurs et les actions mises en place est un autre facteur défavorable important. Si l'on en croit notamment Tiendrebeogo (2004), Locoh et Makdessi (2000) et Bella (1998) ceci serait une conséquence non seulement des moyens limités des Etats africains mais aussi du fait que dans la pratique leurs décideurs n'accordent pas une même importance aux programmes de PF et à ceux portant directement sur le développement économique. D'où la pertinence des actions de repositionnement de la PF engagées dans certains pays africains, parmi lesquelles sont incluses celles de plaidoyer auprès des décideurs et leaders de la communauté.

S'agissant de la disponibilité et l'accessibilité des services de PF, il est clair que si les besoins en PF s'expriment dans un contexte où le nombre de formations sanitaires est important, les formations sanitaires sont à proximité des clients et la PF est intégrée dans les services de santé, ils seront suffisamment satisfaits et la prévalence de la contraception augmentera. Au Rwanda, par exemple, ces facteurs ont joué un rôle important dans l'amélioration des comportements contraceptifs (Belohlav et Nolan, 2013). Un autre facteur important est l'accessibilité économique (MLE, 20I2). En effet, les coûts directs de la contraception (prix des contraceptifs et frais de consultation et de déplacement) et ses coûts indirects (durée de déplacement et temps d'attente à la formation sanitaire) influencent l'utilisation des services de PF.

Par ailleurs, à partir des données de l'EDS réalisée en Tunisie en 1988, Cochrane et Guilkey (1995) ont montré que le facteur le plus déterminant dans l'accroissement de la motivation et du recours effectif des femmes aux moyens contraceptifs est la qualité des services de PF. Au Sénégal, une étude de panel a mis en évidence une relation positive entre le niveau de la qualité des services de PF et l'utilisation de la contraception : les femmes ayant reçu des services de PF de bonne qualité dans les établissements de santé étaient I,3 fois plus susceptibles d'utiliser une méthode contraceptive par rapport aux femmes qui ont reçu des soins de mauvaise qualité (Sanogo et al. 2003). II en est de même aux philippines : la probabilité d'utilisation de contraceptifs modernes était de $55 \%$ pour les clientes ayant reçu des soins de mauvaise qualité, $62 \%$ pour les soins de qualité moyenne, et $67 \%$ pour les soins de haute qualité (Rama Rao et al., 2003). Des conclusions similaires ressortent des études d'impact réalisées au Sénégal, au Bangladesh et en Tanzanie (Koenig et al. 1997 ; Mroz et al. 1999 ; Speizer et Bollen 2000 ; cités par Creel et al., 2002). Elles démontrent que l'amélioration de la qualité des services de santé reproductive encourage l'utilisation des contraceptifs. Celle-ci était notamment plus répandue dans les régions où les clientes avaient l'impression de bénéficier de bons soins que dans les régions ayant des formations sanitaires de moins bonne qualité. La même relation a été observée en Tanzanie dans le cas de certains éléments de la qualité des soins comme l'information donnée aux clientes et la compétence technique des prestataires (ArendsKuenning et Kessy, 2007). Enfin, Blanc et al. (2002) ont constaté que la qualité des services de PF influence aussi l'abandon de l'utilisation de la contraception : les données des EDS de 15 pays qu'ils ont analysées ont révélé qu'en une année, après le début d'utilisation d'une méthode, entre 7 et $27 \%$ des femmes abandonnent la méthode pour des raisons liées à la qualité de l'environnement du service.

\section{Approche demande}

On retrouve ici trois dimensions des BNS en PF, notamment économique, socioculturelle et sociodémographique.

\section{La dimension économique des BNS en PF}

Selon Becker (1960), la fécondité résulte d'un calcul rationnel que font les individus et les décisions d'avoir des enfants visent à maximiser leurs fonctions utilitaires (bénéfice), en tenant compte de la disponibilité des ressources et des coûts à allouer éventuellement à d'autres biens et services concurrentiels. Cette approche fait apparaître que la demande d'enfants a en plus de sa dimension quantitative (leur nombre), une dimension qualitative. La " qualité " d'un enfant se mesure par l'ensemble des dépenses qu'il implique et, de ce fait, augmente avec son niveau d'éducation. Selon cette approche, la faible utilisation de la contraception dans certaines couches sociales des populations africaines est justifiée par le fait que les avantages relatifs aux enfants sont plus importants que les coûts de les élever. L'enfant marginal (l'enfant supplémentaire) 
est désiré par les couples compte tenu de l'utilité (bénéfice) et de la désutilité (coûts) qui s'y rattachent.

Easterlin (1975) a critiqué la conceptualisation de l'offre d'enfant faite par Becker. Selon lui, l'approche de Becker est insuffisante puisqu'elle doit intégrer toutes les dimensions suivantes de la fécondité: la fécondité désirée, la fécondité potentielle ou naturelle et les coûts subjectif et objectif de la régulation de la fécondité. $A$ en croire Easterlin, c'est l'interaction entre la fécondité désirée et la fécondité naturelle qui détermine la régulation de la fécondité et le niveau de ce phénomène. Pour expliquer les tendances de la fécondité dans les pays en développement, une autre approche théorique économique a été proposée par Caldwell (1976). Ce dernier présume notamment que la faible utilisation de la contraception et la forte fécondité observées dans les pays en développement sont justifiées par le fait que dans leurs contextes, où le système de sécurité sociale et de retraite n'est pas fiable, les parents comptent beaucoup sur leurs enfants pour mieux vivre pendant leurs vieux jours. Le changement de la direction des flux de richesses entre générations est donc selon Caldwell (1976) le principal déterminant de la baisse de la fécondité dans ce pays. Pour Caldwell (1982, 1983), l'enfant perd sa valeur économique avec la nucléarisation affective de la famille et cela conduit à la réduction des inégalités de genre.

Enfin, la théorie de stratégie de survie considère la forte fécondité des pauvres comme un moyen rationnel d'améliorer leurs conditions de vie. Selon elle, ils adoptent ce comportement pour maximiser le nombre de personnes économiquement rentables dans leurs ménages. Lorsque les conditions de vie des ménages deviennent difficiles, ces derniers mettent en place des stratégies nécessaires à l'obtention des ressources supplémentaires. Deux modes de production peuvent être utilisés pour $y$ arriver: le premier est basé sur l'économie de subsistance, c'est-à-dire produisant des biens de l'autoconsommation, à l'aide essentiellement des moyens de production faiblement mécanisés ou de la force physique des membres du ménage; le deuxième repose sur le salariat et ce dernier, contrairement au premier, nécessite des arrangements au niveau de la fécondité. Dans le deuxième cas, les formes domestiques de contrôle de la main d'œuvre disparaissent et l'articulation entre la production salariale et domestique se développe (Gregory et Piché, 1985 ; cités par Kourouma, 20l I). Ainsi, l'éducation des enfants et la migration apparaissent comme d'autres moyens d'accession à une condition de vie meilleure.
Dans les études antérieures, pour évaluer la pertinence de l'approche économique dans le contexte africain, le niveau de vie des ménages a été inclus dans les analyses différentielles et explicatives des BNS en PF. II en est ressorti que c'est au Kenya, où des progrès importants ont été réalisés dans le domaine de la PF, qu'une baisse régulière du taux de BNS en PF d'espacement ou de limitation est observée du niveau de vie le plus faible à son niveau le plus élevé (Agwanda et al., 20I5). En revanche, au Ghana, au Sénégal, au Niger et en Guinée l'influence du niveau de vie des ménages sur le phénomène étudié est non significative (GSS, GHS et ICF International, 2015; Ndiaye et Diop, 2012; Hassane et al, 2013 ; Kourouma, 20II).

\section{La dimension socioculturelle des BNS en PF}

La principale critique de la pertinence de l'approche susmentionnée dans le contexte africain est que dans ce dernier les décisions relatives à la fécondité sont prises non seulement par les géniteurs mais aussi par la famille et la société (Ela, 1995 ; Wakam, 2004 ; Vimard et Fassassi, 2007). Le contexte socioculturel africain n'est donc pas homogène. II existe deux cadres de vie, " traditionnel " et "moderne ", dans lesquels se trouvent des individus en interactions permanentes. Dans le premier, une valeur sociale élevée est accordée à l'enfant. Ce qui fait que les décisions d'avoir un enfant n'y dépendent pas de l'individu ou du couple mais de la famille ou la communauté. Pour cela, des normes et valeurs traditionnelles relatives à la fécondité y sont transmises aux individus par les aînés pendant leur socialisation familiale. Dans le deuxième, le contact des individus aux nouvelles idées via l'urbanisation, la scolarisation et l'exposition aux médias leur permet de résister aux pressions de leur milieu socioculturel traditionnel dans le domaine de la fécondité, d'acquérir des connaissances suffisantes dans le domaine de la PF et de ne pas avoir peur des effets secondaires liés à l'utilisation des méthodes contraceptives modernes.

Cependant, les conclusions des études empiriques antérieures n'ont pas convergé dans le même sens. Par exemple, l'urbanisation s'accompagne d'un faible niveau de BNS en PF au Kenya, où l'offre de PF est inégalement répartie sur le territoire (Agwanda et al., 20I5), contrairement au Ghana, où elle est autant élevé en milieu urbain qu'en milieu rural (GSS, GHS et ICF International, 20/5). Concernant le niveau d'instruction, dans les deux pays et dans bien d'autres comme le Cameroun, le Sénégal et le Burkina-Faso, son influence sur le niveau de BNS en PF est significativement négative (Agwanda, Awes et Muia, 
2015; GSS, GHS et ICF International, 2015 ; Fomekong et Tchekanda, 2012 ; Ndiaye et Diop, 2012 ; Sanon et Tapsoba, 20|2), contrairement à ce qui ressort dans quelques rares pays comme le Niger, le Mali et la Guinée (Hassane et al.; 2013 ; CPS/SSDSPF, INSTAT/MPATP, INFO-STAT et ICF International, $20 \mathrm{I} 4$; Kourouma, 20I I). Dans les deux derniers pays, où l'influence de l'exposition aux médias sur les BNS en PF a été évaluée, une relation plutôt positive a été observée (Mariko et al., 2009 ; Kourouma, 20II).

\section{La dimension sociodémographique des BNS en PF}

Selon cette approche, certaines caractéristiques démographiques comme l'âge chronologique, la mortalité des enfants et le nombre d'enfants survivants influencent les BNS en PF. L'étude d'Osaka (2008, cité par Kourouma, 20I I) au Kenya a mis en évidence une diminution des besoins non satisfaits avec l'augmentation de l'âge de la femme au moment de l'enquête. Dans une série d'études réalisées en Afrique subsaharienne à partir des EDS, Akoto et ses collègues (2002) ont montré aussi que l'importance des BNS en espacement décroît avec l'âge, contrairement à celle des BNS en limitation, qui augmente avec l'âge. Un résultat similaire a été, dans une certaine mesure, mis en évidence au Bénin par Sanni (20I I) et au Mali par Mariko et al. (2009). Toutefois, dans les pays d'Afrique subsaharienne, où l'âge et la parité atteinte sont généralement liées, leur prise en compte simultanée dans les modèles multivariés devrait permettre de savoir si l'effet de génération est aussi important lorsqu'on analyse les BNS en PF, les jeunes femmes ont notamment vécu dans des conditions sanitaires et technologiques meilleures que les plus âgées et seraient plus exposées et plus sensibles que ces derniers aux campagnes sur la PF (Akoto et al., 2002). S'agissant de la mortalité des enfants, Khan et al. (2008, cités par Kourouma, 20II) ont constaté en Ouganda que le nombre d'enfants survivants influence négativement les BNS en PF. En revanche, en Guinée, Kourouma (20II) a mis en évidence une relation positive entre les deux variables, quels que soient le milieu de résidence et le type de BNS en PF.

\section{Approche globale des BNS en PF}

Bien que les approches susmentionnées soient toutes pertinentes dans le contexte africain, aucune étude empirique antérieure n'a utilisé l'approche intégrant l'offre et la demande dans la recherche des déterminants des BNS en PF. A cet effet, pour pallier à cette limite importante, nous avons inscrit notre étude dans une perspective explicative globale, selon laquelle, l'offre des services de PF et les caractéristiques d'identification sociale de la femme sont associés à ses BNS en PF.

Parmi ces dernières, on retrouve notamment celles relatives au milieu socioculturel traditionnel, à savoir le cadre dans lequel les normes et valeurs traditionnelles en matière de fécondité et PF ont été transmises à la femme par sa famille et intériorisées par elle, à la modernité culturelle et à la modernité économique. En effet, pour mieux comprendre les comportements contraceptifs, dans le contexte africain, il est important de s'intéresser non seulement aux poids des traditions mais aussi aux effets des distances que prennent les individus vis-àvis de ces dernières aux plans culturel et économique.

Dans le cas spécifique de la femme, notre approche se démarque aussi des autres par sa prise en compte au niveau de la demande d'une variable rendant compte de l'effet de son statut, à savoir son occupation, et d'une autre relative aux inégalités de genre. En effet, nous présumons que dans le même contexte, l'exercice par la femme d'une activité dans le secteur moderne de l'économie la motive soit à résister aux pressions que la famille et la société exercent sur elle dans le domaine de la fécondité, soit à être défavorable aux rapports inégalitaires de genre et même à être autonome dans la prise des décisions dans le domaine de la fécondité. De même, nous présumons que l'exercice par la femme d'une activité dans le secteur moderne de l'économie lui permet d'interagir avec d'autres femmes bien informées et éduquées dans le domaine de la PF et expérimentées dans ce domaine.

La recherche qualitative réalisée par Gilda et al. (2016) témoigne de la pertinence de l'approche globale des BNS en PF. Ils ont notamment constaté que la non connaissance des méthodes contraceptives, l'inaccessibilité aux sources d'approvisionnement en contraceptifs et le coût élevé des contraceptifs sont les facteurs les plus importants des BNS en PF en Afrique de l'Ouest et en Afrique Centrale que dans d'autres régions du monde.

\section{Méthodologie}

Sources des données

Les données utilisées dans cette étude sont issues des EDS réalisées au Mali en 1995-96, 2006 et 2012-13. Les deux premières sont représentatives de l'ensemble des régions du pays et de Bamako. En revanche, l'EDS de 2012-I3 n'a pas couvert le Nord du Mali, à savoir Tombouctou, Gao et Kidal, à cause de l'insécurité. De même, la région de Mopti a été partiellement couverte pour la même raison. Leurs échantillons sont tous basés sur un sondage par grappes stratifié à deux degrés. Ainsi, contrairement aux EDS 1995-96 et 2006, qui avaient onze domaines 
d'études composés de chacune des huit régions (Kayes, Koulikoro, Sikasso, Ségou, Mopti, Gao, Tombouctou et Kidal, du district de Bamako, du milieu urbain et du milieu urbain et du milieu rural du Mali, l'EDS de 20/2-13 comprend huit domaines constitués de cinq premières régions mentionnées dans le cas de deux autres EDS, du district de Bamako et de l'ensemble de leur milieu urbain et rural.

Les trois EDS ont été réalisées selon un plan de sondage comportant plusieurs grappes en milieux urbain et rural, dans lesquels des ménages ont été sélectionnés au hasard et dans ces ménages des femmes âgées de $15-49$ ans ont été enquêtées : à l'EDS de 1995-96, 3509 en milieu urbain et 6/95 en milieu rural ; à l'EDS de 2006, respectivement $5 / 43$ et 9440 ; à l'EDS de 2012-I3, respectivement 3262 et 7/62. Bien que la part de l'échantillon national des ménages du Mali non inclus dans l'EDS 2012-13 représente moins de $10 \%$ (CPS/SSDSPF, INSTAT/MPATP, INFO-STAT et ICF International, 20I4), pour que les trois EDS soient comparables, les informations concernant les trois régions du Nord

Tableau I: Disponibilité des informations sur les variables opérationnelles des concepts

\begin{tabular}{|c|c|c|c|c|}
\hline Concepts & Variables & EDS 1995-96 & EDS 2006 & EDS 2012-13 \\
\hline \multirow[t]{2}{*}{ Offre des services de PF } & $\begin{array}{l}\text { Visites à domiciles des Agents de Santé } \\
\text { Communautaire (ASC) au cours du dernier } \\
\text { mois précédant l'enquête }\end{array}$ & ND & $\mathrm{D}$ & $\mathrm{D}$ \\
\hline & $\begin{array}{l}\text { Réception par l'enquêtée des informations sur } \\
\text { la PF auprès d'un prestataire des soins lorsqu'il } \\
\text { l'a consultée dans un centre de santé pendant la } \\
\text { même période de référence }\end{array}$ & $\mathrm{D}$ & $\mathrm{D}$ & $\mathrm{D}$ \\
\hline \multirow[t]{2}{*}{ Milieu socioculturel traditionnel } & Ethnie & $\mathrm{D}$ & D & D \\
\hline & Religion & $\mathrm{D}$ & $\mathrm{D}$ & $\mathrm{D}$ \\
\hline \multirow{8}{*}{ Modernité culturelle } & Milieu de résidence à l'enfance & $\mathrm{D}$ & $\mathrm{D}$ & ND \\
\hline & Milieu de résidence à l'enquête & D & D & D \\
\hline & Niveau d'instruction de la femme & $\mathrm{D}$ & D & $\mathrm{D}$ \\
\hline & Niveau d'instruction du conjoint & $\mathrm{D}$ & $\mathrm{D}$ & $\mathrm{D}$ \\
\hline & Occupation de la femme & $\mathrm{D}$ & D & D \\
\hline & Fréquence d'écoute de la radio & $\mathrm{D}$ & $\mathrm{D}$ & $\mathrm{D}$ \\
\hline & Fréquence d'exposition à la TV & $\mathrm{D}$ & D & D \\
\hline & Fréquence de lecture des journaux/Magazines & $\mathrm{D}$ & $\mathrm{D}$ & $\mathrm{D}$ \\
\hline \multirow{2}{*}{ Modernité économique } & Niveau de vie & ND & D & D \\
\hline & Occupation du conjoint & $\mathrm{D}$ & $\mathrm{D}$ & $\mathrm{D}$ \\
\hline Caractéristiques & Age & $\mathrm{D}$ & $\mathrm{D}$ & $\mathrm{D}$ \\
\hline \multirow[t]{5}{*}{ sociodémographiques } & Age au premier mariage & $\mathrm{D}$ & D & D \\
\hline & Nombre d'enfants décédés & $\mathrm{D}$ & $\mathrm{D}$ & $\mathrm{D}$ \\
\hline & Nombre d'enfants nés vivants & D & $\mathrm{D}$ & $\mathrm{D}$ \\
\hline & Nombre d'enfants survivants & $\mathrm{D}$ & $\mathrm{D}$ & D \\
\hline & Nombre de partenaires sexuels & ND & $\mathrm{D}$ & ND \\
\hline \multirow[t]{3}{*}{ Rapports de genre } & Discussion au sein du couple sur la PF & D & D & ND \\
\hline & Attitude du mari vis-à-vis de la PF & $\mathrm{D}$ & $\mathrm{D}$ & ND \\
\hline & Attitude de la femme vis-à-vis de la PF & $\mathrm{D}$ & $\mathrm{D}$ & ND \\
\hline \multirow{3}{*}{ BNS en PF } & Etat matrimonial & $\mathrm{D}$ & $\mathrm{D}$ & $\mathrm{D}$ \\
\hline & Utilisation actuelle de la contraception & $\mathrm{D}$ & D & $\mathrm{D}$ \\
\hline & Préférences en matière de fécondité & $\mathrm{D}$ & $\mathrm{D}$ & $\mathrm{D}$ \\
\hline
\end{tabular}

Notes : ND=Non disponible ; $\mathrm{D}=$ Disponible.

(Tombouctou, Gao et Kidal) n'ont pas été prises en compte dans les EDS de 1995-96 et 2006. Comme le montre le tableau I ci-haut, on retrouve dans les sources des données utilisées les informations sur les variables opérationnelles de nos principaux concepts.

\section{Variables}

\section{Variable dépendante}

C'est le fait d'avoir des BNS en PF ou non. Pour mesurer ces derniers à partir des EDS, nous avons utilisé la méthode suivante de Westoff et Bankole (1995):
- On a considéré dans la population de base l'ensemble des femmes en union avec un homme au moment de l'enquête et fertiles;

- On a sélectionné les femmes qui n'utilisent pas actuellement la contraception;

- On a séparé les femmes enceintes ou en aménorrhée post-partum de celles qui sont ni enceintes ni en aménorrhée post-partum ;

On a ensuite classé les femmes enceintes ou en aménorrhée post-partum en trois groupes: celles dont la grossesse a été désirée, celles dont la grossesse a été mal planifiée ou mal calculée et celles qui ne voulaient pas du tout tomber enceintes. Le deuxième groupe est celui des femmes ayant des 
BNS en espacement des naissances et le troisième celui des femmes ayant des BNS en limitation des naissances.

- On a enfin classé les femmes qui sont ni enceintes ni en aménorrhée post-partum en deux groupes: les femmes fécondes et celles stériles. Chez les premières on a constitué trois sous-groupes: celles qui veulent avoir d'enfants bientôt, celles qui veulent en avoir plus tard et celles qui ne veulent plus en avoir. Le deuxième sous-groupe est celui des femmes ayant des BNS en espacement des naissances et le troisième celui des femmes ayant des BNS en limitation des naissances.

Pour nous rassurer que la variable a été bien construite, nous l'avons comparée à celle créée par ICF International et contenue dans la base des données considérée.

Ainsi, nous avons considéré dans cette étude trois formes des BNS en PF ayant chacune deux modalités: I) quand la femme a des BNS en PF pour espacer les naissances, 0) sinon ; I) quand elle a des BNS en PF pour limiter les naissances, 0 ) sinon ; I) quand elle a des BNS en PF (quel que soit le type de besoins), 0) sinon. La définition des BNS considérée ici a conduit à ce que sa mesure porte seulement sur les femmes actuellement en union.

\section{Variables indépendantes}

Nous avons inclus dans l'ensemble de ces variables, au niveau de l'offre des services de PF, deux indicateurs de la qualité des services de PF, à savoir les visites à domiciles des ASC dans le cadre des services de $\mathrm{PF}$ et la réception des informations sur la $\mathrm{PF}$ dans les centres de santé lors des dernières consultations médicales. Nous présumons ici que les deux variables influencent négativement la prévalence des BNS en PF. On retrouve aussi dans l'ensemble des variables indépendantes, deux indicateurs du milieu socioculturel traditionnel, l'ethnie et la religion. La variation régionale non significative des BNS de PF observée par CPS/SSDSPF, INSTAT/MPATP, INFOSTAT et ICF-International (20/4) traduit la même relation dans le cas de la variation ethnique. En revanche, nous présumons ici une variation du risque de BNS en PF selon la religion, les femmes chrétiennes ou animistes étant moins enclines que les musulmanes à exprimer les BNS en PF, compte tenu du fait que les changements qui ont eu lieu au Mali a plus entraîné les premières que les dernières à moins s'attacher aux modèles culturels traditionnels de PF. Les distributions des fréquences des variables susmentionnées sont présentées au tableau 2.

Le degré de modernité culturelle est une autre variable indépendante. C'est une variable composite que nous avons créée à l'aide de l'Analyse en Composantes Principales (ACP) sur les différents indicateurs de la modernité culturelle, à savoir le milieu de résidence à l'enfance, le milieu de résidence à l'enquête, le niveau d'instruction de la femme, le niveau d'instruction du conjoint, l'occupation de la femme, la fréquence d'écoute de la radio, la fréquence d'exposition à la TV et la fréquence de lecture des journaux/Magazines (cf. annexe $\mathrm{n}^{\circ} \mathrm{I}$ ). En plus, on retrouve deux indicateurs de la modernité économique : le niveau de vie du ménage et l'occupation du conjoint. En nous référant aux études antérieures, nous présumons que le degré de modernité culturelle influence négativement le risque de BNS en PF. Il en est de même du niveau de vie. S'agissant de l'occupation du conjoint, l'union d'une femme avec un homme économiquement aisé la prédispose à un faible risque de BNS en PF.

Tableau 2: Distributions des fréquences des variables indépendantes qualitatives

\begin{tabular}{|c|c|c|c|c|c|c|}
\hline \multirow[t]{2}{*}{ Variables indépendantes et modalités } & \multicolumn{2}{|c|}{ EDS I995-96 } & \multicolumn{2}{|c|}{ EDS 2006} & \multicolumn{2}{|c|}{ EDS 20I2-13 } \\
\hline & $\mathrm{N}$ & $\%$ & $\mathrm{~N}$ & $\%$ & $\mathrm{~N}$ & $\%$ \\
\hline \multicolumn{7}{|l|}{ A reçu les visites des ASC } \\
\hline Oui & ND & ND & $|45|$ & $\mathrm{II}, 4$ & 1029 & 11,7 \\
\hline Non & & & 11278 & 88,6 & 7770 & 88,3 \\
\hline Ensemble & & & 12729 & 100,0 & 8799 & 100,0 \\
\hline \multicolumn{7}{|l|}{ Prestataires des soins lui ont parlé de la PF } \\
\hline Oui & 552 & 23,0 & 1219 & 33,0 & 1074 & 47,0 \\
\hline Non & 1855 & 77,0 & 2474 & 67,0 & 1209 & 53,0 \\
\hline Ensemble & 2407 & 100,0 & 3693 & 100,0 & 2283 & 100,0 \\
\hline \multicolumn{7}{|l|}{ Ethnie } \\
\hline Mandingue & 4049 & 50,0 & 7217 & 56,7 & 4751 & 54,0 \\
\hline Soudanienne & 1036 & 12,8 & 1120 & 8,8 & 1056 & 12,0 \\
\hline Voltaïque & 745 & 9,2 & 1274 & 10,0 & 889 & 10,1 \\
\hline Nomade & 1393 & 17,2 & 2380 & 18,7 & 1522 & 17,3 \\
\hline Autres ethnies & 874 & 10,8 & 738 & 5,8 & 581 & 6,6 \\
\hline Ensemble & 8097 & 100,0 & 12729 & 100,0 & 8799 & 100,0 \\
\hline \multicolumn{7}{|l|}{ Religion } \\
\hline Musulmane & 7311 & 90,3 & 11634 & 91,4 & 8157 & 92,7 \\
\hline Chrétienne & 235 & 2,9 & 344 & 2,7 & 361 & 4,1 \\
\hline Animiste & 421 & 5,2 & 255 & 2,0 & 87 & 1,0 \\
\hline Autres & 130 & 1,6 & 496 & 3,9 & 194 & 2,2 \\
\hline
\end{tabular}




\begin{tabular}{|c|c|c|c|c|c|c|}
\hline Ensemble & 8097 & 100,0 & 12729 & 100,0 & 8799 & 100,0 \\
\hline \multicolumn{7}{|c|}{ Degré de modernité culturelle } \\
\hline Très faible & 2116 & 27,5 & 2991 & 24,8 & 1908 & 25,4 \\
\hline Faible & 1764 & 22,9 & 3535 & 29,3 & 1852 & 24,7 \\
\hline Moyen & 1891 & 24,6 & 2560 & 21,2 & $|87|$ & 24,9 \\
\hline Elevé & 1924 & 25,0 & 2997 & 24,7 & 1878 & 25,0 \\
\hline Ensemble & 7695 & 100,0 & 12063 & 100,0 & 7509 & 100,0 \\
\hline \multicolumn{7}{|l|}{ Niveau de vie } \\
\hline Très pauvre & ND & ND & 2812 & 22,1 & 1770 & 20,1 \\
\hline Pauvre & & & 2648 & 20,8 & 1777 & 20,2 \\
\hline Moyen & & & 2597 & 20,4 & 1777 & 20,2 \\
\hline Riche & & & 2495 & 19,6 & 1777 & 20,2 \\
\hline Très riche & & & 2177 & 17,1 & 1698 & 19,3 \\
\hline Ensemble & & & 12729 & 100,0 & 8799 & 100,0 \\
\hline \multicolumn{7}{|l|}{ Occupation du conjoint } \\
\hline Inactifs & $13 \mid$ & $\mathrm{I}, 6$ & $(-)$ & $(-)$ & $\mathrm{NU}$ & NU \\
\hline Cadres/employés & 424 & 5,2 & 501 & 4,1 & & \\
\hline Commerçants & 712 & 8,8 & 1805 & 14,9 & & \\
\hline Agriculteurs & 5460 & 67,3 & 8165 & 67,3 & & \\
\hline Services & 933 & 11,5 & $(-)$ & $(-)$ & & \\
\hline Ouvriers & 433 & 5,3 & 1386 & $i, 4$ & & \\
\hline Autres & $(-)$ & $(-)$ & 283 & 2,3 & & \\
\hline Ensemble & 8094 & 100,0 & $|2| 4 \mid$ & 100,0 & & \\
\hline \multicolumn{7}{|c|}{ Discussion au sein du couple sur la PF } \\
\hline Pas du tout & 5287 & 65,3 & 7961 & 62,7 & ND & ND \\
\hline Une ou deux fois & 1506 & 18,6 & 2443 & 19,2 & & \\
\hline Souvent & 1304 & 16,1 & 2303 & 18,1 & & \\
\hline Ensemble & 8097 & 100,0 & 12706 & 100,0 & & \\
\hline \multicolumn{7}{|c|}{ Attitudes du couple vis-à-vis de la PF } \\
\hline Approuve & 1968 & 47,1 & 3455 & 42,9 & & \\
\hline Désapprouve & 1055 & 25,2 & 2349 & 29,1 & ND & ND \\
\hline Femme approuve seule & 1061 & 25,4 & 2111 & 26,2 & & \\
\hline Conjoint approuve seul & 97 & 2,3 & 147 & $\mathrm{I}, 8$ & & \\
\hline Ensemble & 4182 & 100,0 & 8062 & 100,0 & & \\
\hline
\end{tabular}

Notes : ND=Non disponible. $\mathrm{D}=$ Disponible. $\mathrm{NU}=$ non utilisée à cause des erreurs d'observation qui ont été commises sur cette variable pendant la collecte des données ; pour cela on ne retrouve pas dans la base des données les étiquettes des différents codes. (-) modalité inexistante ou très faiblement représentée.

Plusieurs variables sociodémographiques, notamment l'âge au premier mariage, le nombre d'enfants survivants et la proportion d'enfants décédés font également partie des variables indépendantes. Dans le contexte étudié, la corrélation entre les deux dernières variables est faible $(r=-0,22$ en 19951996, $-0,19$ en 2006 et $-0,12$ en 2012-13) (résultats non présentés). En revanche, celle entre l'âge, la parité atteinte et le nombre d'enfants survivants est élevée $(r>0,6)$ (résultats non présentés). C'est pourquoi, pour éviter le problème de multicollinéarité, l'âge et la parité atteinte ont été exclus de l'ensemble des variables indépendantes. De même ici, en nous référant aux études antérieures, nous émettons les hypothèses suivantes : i) le risque de BNS en PF est plus élevé chez les femmes ayant précocement contracté leurs unions que chez celles qui les ont tardivement contractées ; ii) ce risque diminue avec la proportion d'enfants décédés et augmente avec le nombre d'enfants survivants.

Enfin, l'influence des rapports de genre est appréhendée dans cette étude par la discussion au sein du couple sur la PF et les attitudes du couple visà-vis de la PF. Les rapports égalitaires de genre, à savoir le dialogue au sein du couple sur la PF et le fait que les deux conjoints approuvent la PF, réduisent le risque de $\mathrm{BNS}$ en $\mathrm{PF}$.

\section{Méthodes statistiques d'analyse}

Pour vérifier toutes nos hypothèses, les méthodes bivariées et celles multivariées ont été utilisées, mais seulement les résultats issus des dernières ont été présentés et interprétés ici. Comme chacune des formes de BNS a deux modalités: a les BNS en PF pour l'espacement des naissances ou non, les BNS en PF pour la limitation des naissances ou non, le total des BNS en PF ou non, les modèles multivariés de régression logistique binaire ont été utilisés. Nous n'avons pas présenté ici les coefficients de régression $(\beta)$, mais les odds ratios (c.-à-d. les exponentiels de $\beta$ ) puisqu'ils sont plus faciles à interpréter. La modalité de référence a été choisie pour chacune des variables indépendantes. En 2006, compte tenu du problème de multicollinéarité observé entre le niveau 
de vie et l'occupation du conjoint, nous avons produit deux modèles : dans l'un on retrouve la première variable et dans l'autre la deuxième.

Au-delà de l'examen des odds ratios permettant d'identifier les facteurs associés aux comportements étudiés et de caractériser les femmes qui en sont les plus concernées, nous avons procédé comme Kobiane (20I4) à une hiérarchisation des variables en utilisant le Khi-deux du modèle, ce qui permet de connaître les facteurs sur lesquels il faudra mettre la priorité. En clair, cette hiérarchisation des variables s'est faite à partir du calcul de la contribution $\left(C_{i}\right)$ de chacune d'elles à l'explication du phénomène étudié, mesurée par la statistique du Khi-deux du modèle. La contribution de la variable indépendante $X_{i}$ est donnée par la formule suivante:

$C_{i}=\frac{\chi_{\mathrm{f}}^{2}-\chi_{\mathrm{f}-\mathrm{i}}^{2}}{\chi_{\mathrm{f}}^{2}}$ (où $\chi_{\mathrm{f}}^{2}$ est le Khi-deux du modèle global et $\chi_{\mathrm{f}-\mathrm{i}}^{2}$ est le Khi-deux sans la variable indépendante considérée).

En clair, la hiérarchisation a conduit à ordonner les variables indépendantes en fonction du pourcentage de Khi-deux qu'elles apportent à l'explication de la variable dépendante considérée. Les données ont été analysées sous le logiciel SPSS.

\section{Résultats}

Facteurs associés aux BNS en PF et leur hiérarchisation

En 1995-96, on retrouve les facteurs associés aux BNS en PF uniquement dans les trois dimensions de la demande de PF considérées ici (tableau 3: colonnes I, 2 et 3). En revanche, en 2006 et 20I2-I3, on les retrouve aussi parmi les caractéristiques de l'offre de PF (tableau 3: colonnes 4, 6, 8, 9, I0, II et I2). En 1995-96, les facteurs expliquant plus de $60 \%$ de la variation du risque des BNS pour espacer les naissances et qui sont aux trois premiers rangs dans la hiérarchie des facteurs sont la proportion d'enfants décédés, le nombre d'enfants survivants et les attitudes du couple vis-à-vis de la PF (tableau 4: colonne 5). En 2006, on retrouve à la place de la première variable la réception des visites des ASC. En 20I2-I3, la réception de l'information sur la PF dans les centres de santé intègre l'ensemble des facteurs les plus importants de la même variable dépendante ainsi que le degré de modernité culturelle et le niveau de vie (tableaux 5 à 7: colonne $5)$. 
African Population Studies Vol.33, No. I, Feb. 2019

Tableau 3: Résultats des modèles saturés de régression logistique binaire sur les BNS en PF

\begin{tabular}{|c|c|c|c|c|c|c|c|c|c|c|c|c|}
\hline \multirow[t]{2}{*}{ Variables et modalités } & \multicolumn{3}{|c|}{ EDS 1995-96 } & \multicolumn{6}{|c|}{ EDS 2006} & \multicolumn{3}{|c|}{ EDS 2012-13 } \\
\hline & $\begin{array}{l}\text { Espacer } \\
\text { (I) }\end{array}$ & $\begin{array}{l}\text { Limiter } \\
\text { (2) }\end{array}$ & $\begin{array}{l}\text { Total } \\
\text { (3) }\end{array}$ & $\begin{array}{l}\text { Espacer } \\
(4)\end{array}$ & (5) & $\begin{array}{l}\text { Limiter } \\
(6)\end{array}$ & (7) & $\begin{array}{l}\text { Total } \\
\text { (8) }\end{array}$ & (9) & $\begin{array}{l}\text { Espacer } \\
(10)\end{array}$ & $\begin{array}{l}\text { Limiter } \\
\text { (II) }\end{array}$ & $\begin{array}{l}\text { Total } \\
(12)\end{array}$ \\
\hline Offre des services de PF & & & & & & & & & & & & \\
\hline $\begin{array}{l}\text { Reçu les visites des ASC? } \\
\text { Oui }\end{array}$ & ND & ND & ND & $0,873 * *$ & 0,901 & $1,254 * *$ & 1,167 & 0.967 & 0,965 & 1.003 & | $449 \% * * *$ & 1,137 \\
\hline $\begin{array}{l}\text { Non } \\
\text { Prestataire lui a parlé de PF dans le } \\
\text { centre de santé ? }\end{array}$ & & & & & $(r)$ & $(r)$ & (r) & (r) & (r) & (r) & $(r)$ & $(r)$ \\
\hline $\begin{array}{l}\text { Oui } \\
\text { Non }\end{array}$ & $\begin{array}{l}0,936 \\
(r)\end{array}$ & $\begin{array}{l}0,805 \\
(r)\end{array}$ & $\begin{array}{l}0,883 \\
(r)\end{array}$ & $\begin{array}{l}0,943 \\
(r)\end{array}$ & $\begin{array}{l}0,889 \\
(r)\end{array}$ & $\begin{array}{l}0,868 \\
(r)\end{array}$ & $\begin{array}{l}0,870 \\
(r)\end{array}$ & $\begin{array}{l}0,847 * * \\
(r)\end{array}$ & $\begin{array}{l}0,864 * \\
(r)\end{array}$ & $\begin{array}{l}0,688 * * * \\
(r)\end{array}$ & $\begin{array}{l}0,690 * \\
(r)\end{array}$ & $\begin{array}{l}0,653^{* * * *} \\
(\mathrm{r})\end{array}$ \\
\hline $\begin{array}{l}\text { N'a pas fréquenté un centre de santé } \\
\text { Milieu socioculturel traditionnel } \\
\text { Ethnie }\end{array}$ & $0,768 * * *$ & I,247* & $0,847 * * * *$ & $0,885 * * *$ & $0,886 * *$ & $1,253 * *$ & I,199* & 0,927 & 0,939 & 0,878 & 1,115 & 0,927 \\
\hline Mandingue & (r) & (r) & $(r)$ & $(\mathrm{r})$ & $(r)$ & $(r)$ & $(r)$ & $(r)$ & $(r)$ & $(r)$ & $(r)$ & $(r)$ \\
\hline Soudanienne & 0,996 & 0,766 & 0,940 & $0,866 * *$ & 0,880 & $0,772 * *$ & $0,722 * * * *$ & $0,817 * * * *$ & $0,8 \mid 8 * * *$ & 1,168 & 0,947 & 1,117 \\
\hline Voltaïque & $0,786 * *$ & 1,113 & 0,846 & $0,888 * *$ & 0,923 & $0,758 * *$ & $0,739 * * * *$ & $0,835 * * *$ & $0,859 * *$ & 1,067 & 0,868 & 1,001 \\
\hline Nomade & 0,939 & 1,074 & 0,960 & 0,936 & 0,919 & $0,640 * * *$ & $0,680 * * *$ & $0,836 * * *$ & $0,838 * * *$ & 1,005 & 1,008 & 1,005 \\
\hline Autres & 0,894 & 0,929 & 0,890 & $1,257^{* * * *}$ & $1,200^{* *}$ & $0,649 * * *$ & $0,676^{* * * *}$ & 1,080 & 1,055 & 1,052 & $1,720 * * *$ & $1,258 * *$ \\
\hline Religion & & & & & & & & & & & & \\
\hline Musulmane & $(r)$ & (r) & $(r)$ & $(r)$ & $(r)$ & $(r)$ & $(r)$ & $(r)$ & $(r)$ & $(r)$ & $(r)$ & $(r)$ \\
\hline Chrétienne & 0,999 & 0,841 & 0,939 & 1,090 & 1,168 & 1,43।* & 1,462* & 1,222 & 1,260 & $0,658 * *$ & 1,048 & 0,735 ** \\
\hline Animiste & $0,640 * * *$ & $0,559 * *$ & $0,598 * * *$ & 0,812 & 0,900 & 1,796**** & I,732**** & 1,115 & I,083 & 0,986 & 1,460 & 1,169 \\
\hline Autres & $1,636 * *$ & 1,384 & $1,634 * *$ & 1,081 & 1,124 & 1,183 & 1,125 & 1,129 & 1,123 & 1,119 & 0,623 & 0,939 \\
\hline Degré de modernité culturelle & & & & & & & & & & & & \\
\hline Très faible & $(r)$ & $(r)$ & $(r)$ & $(r)$ & $(r)$ & $(r)$ & $(r)$ & $(r)$ & $(r)$ & $(\mathrm{r})$ & $(\mathrm{r})$ & $(r)$ \\
\hline Faible & $1.179 *$ & 1,003 & $1,142 *$ & 1.131 **** & $1,114 *$ & 1.035 & 1.064 & $1,114 * *$ & $1,104 *$ & $1,180 *$ & 1,113 & $1,185 * 6$ \\
\hline Moyen & $1,209 * *$ & $1,385 * * * *$ & $1,282 * * * *$ & , 277 **** & I, $191 * * * *$ & 0,860 & 0,902 & I, 173** & $1,121 * *$ & $1,376 * * *$ & 1,029 & $|, 30| * * * *$ \\
\hline Elevé & 1,152 & 1,203 & $1,171^{*}$ & I,476**** & $|, 27| * * *$ & 1,029 & 1,2195 & I,406*** & I,293*** & 1,383**** & 0,954 & $1,292 * * *$ \\
\hline Degré de modernité économique & & & & & & & & & & & & \\
\hline Niveau de vie & ND & ND & ND & & NU & & NU & & NU & & & \\
\hline Très pauvre & & & & $(r)$ & & (r) & & (r) & & (r) & $(\mathrm{r})$ & $(r)$ \\
\hline Pauvre & & & & 0,912 & & , $265 * *$ & & 1,019 & & 0,906 & 1,186 & 0,993 \\
\hline Moyen & & & & $0,817 * * *$ & & $1,614 * * *$ & & 0,954 & & 1,115 & 1,039 & 1,104 \\
\hline Riche & & & & $0,74 I^{* * * *}$ & & $1,330 * *$ & & $0,846 * * *$ & & 1,067 & 0,877 & 1,018 \\
\hline Très riche & & & & $0,752 * * *$ & & $1,639 * * *$ & & 0,894 & & $0,787 * *$ & 1,053 & $0,827 * *$ \\
\hline $\begin{array}{l}\text { Occupation du conjoint } \\
\text { Inactifs }\end{array}$ & & & & ND & & ND & & ND & & ND & ND & ND \\
\hline Cadres/employés & 1,153 & 0,733 & 1,098 & & - & & - & & - & & & \\
\hline Commerçants & $0,662 * * *$ & 0,859 & $0,680 * * * *$ & & $0,681 * * * *$ & & 1,069 & & $0,728 * * *$ & & & \\
\hline Agriculteurs & 0,968 & 1,197 & 1,017 & & ।,119* & & $0,7 \mid 3 * * *$ & & 1,026 & & & \\
\hline Services & (r) & $(r)$ & $(r)$ & & $(r)$ & & $(r)$ & & $(r)$ & & & \\
\hline Ouvriers & 0,980 & $1,510 * *$ & 1,108 & & 1,679** & & 0,388 & & $1,506 * *$ & & & \\
\hline Autres & 0,915 & 1,037 & 0,943 & & 1,058 & & $1,627 * * * *$ & & $1,190 * * *$ & & & \\
\hline & - & - & - & & $1,265 *$ & & 0,945 & & $1,238 *$ & & & \\
\hline
\end{tabular}


Tableau 3: Suite et fin.

\section{Variables et modalités}

$$
\text { EDS 1995-96 }
$$

$$
\begin{aligned}
& \text { Espace } \\
& (1)
\end{aligned}
$$

Limite
$(2)$

Limiter
(2)

Tota
(3)

Total
(3)

EDS 2006

(5)

Limiter
$(6)$

(7)

$\begin{array}{ll}(r) & (r) \\ 0,904 & 1,190 * * *\end{array}$

$0,823 * * *$

$0,732 * * * *$

1,037

$0,858 * * \quad 0,825$

$0,817^{*} \quad 0,620 * *$

$19-24$
$25+$

Proportion enfants décédés (en

$\%)$

0
$7-24$
$25-39$

25-39

$60+$

Nombre enfants survivants

0

I

3
4
5
$6+$

$6+$

Rapports de genre

$2,878 * * *$

$3,363 * * * 3$

, $925 * * *$

(r)

0,383

$2,182 * * *$

1,149

$0,267 * * *$

$0,446 * *$

$0,537 * *$ *

$2,182 * * *$

$2,804 * * *-1,590 * * *$

$1,938 * * *$

(r)

$1,800 * * *$

$$
\text { (r) }
$$

$0,349 * * * \quad 0,755$

0,009

$0,005 * * *$

0,037 ****

$0,165 * * *$

$0,649 * * *$

$0,697 * * *$

$0,730 * * *$

(r)

$$
\text { (r) }
$$

I,770****

I,847****

$1,689 * * *$

(r)

$(\mathrm{r})$
$1,180 * * *$
1,040
1,055
1,140
$1,633 * * *$
$1,701 * * *$
$1,632 * * *$
$1,868 * * *$
$(\mathrm{r})$

$(r)$
$0,741 * * * *$

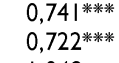

1,048

0,978

$0,199 * * *$

0,275 ****

$0,443 * * *$

(r)

$0,770 \quad 0,005^{* * * *}$

1,709****

I,830****

$1,715^{* * * *}$

(r)

$0,017 * * *$

$0,041 * * * * 1$

$0,163 * * *$

0,365 ****

(r)

(r)

\section{$(r)$
$0,736 * *$}

$0,689 * * *$

1,031
0,938

$0,189 * * *$

$0,266 * * *$

$0,344 * * * *$

0,426
$(r)$

$0,005 * * * \quad 0,185 * *$

$0,015 * * *$

$0,040 * * * * 1$

$0,069 * * *$

$0,165 * * *$

(r)

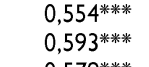

$0,579 * * *$

$0,698 * * * 1$

(r)

Discussion au sein du couple sur
la PF

(r)

(r) $\quad(r)$

(r) $\quad(r)$

(r)

(r)

$(r)$
0,979

$1,151 * *$
$0,875 * *$

I,166***

1,020
1,020

0,988

1,668****

I,564****

(r) $2,725 * * *$

Approuve

Désapprouve

Femme seule

$1,872 * * *$

0,926

(r)
$2,237 * * * *$

(r)
$2,077 * * * *$

(r) $679 * * *$

1,679****
0,795

11763

I,672*** 1,645***

(r)

1,70 I****

0,823

$(\mathrm{r})$
$2,442 * * * *$

$2,442 * * * *$

11763

$1,661 * * *$

(r) $\quad|, 77| * * *$

2,478****

$4,402 * * *$

| 158 |,59***

(r)
$2,038 * * * * 1$

1,401*

11763
517,11 ****

$\stackrel{(r)}{\stackrel{(r)}{1,110^{*}}} \quad \stackrel{\mathrm{l}}{\mathrm{l}, 117^{* *}}$

1,1 17**

I,785****

(r) $2,063 * * *$

I,453**

11582

$\begin{array}{ll}582 & 7424 \\ 122,06 * *\end{array}$

\begin{tabular}{|c|c|c|c|c|c|c|c|c|c|c|c|c|c|c|c|}
\hline \multirow[t]{2}{*}{ Variables } & \multicolumn{5}{|l|}{ Espacer } & \multicolumn{5}{|l|}{ Limiter } & \multicolumn{5}{|l|}{ Total } \\
\hline & $\begin{array}{l}X_{f}^{2} \\
(I)\end{array}$ & $\begin{array}{l}X_{f-1}^{2} \\
(2)\end{array}$ & $\begin{array}{l}C_{i} \text { en \% } \\
\text { (3) }\end{array}$ & $\begin{array}{l}f_{i} \text { en \% } \\
\text { (4) }\end{array}$ & $\begin{array}{l}\text { Rang } \\
\text { (5) }\end{array}$ & $\begin{array}{l}X_{f}^{2} \\
(6)\end{array}$ & $\begin{array}{l}X_{f-1}^{2} \\
(7)\end{array}$ & $\begin{array}{l}C_{i} \\
(8)\end{array}$ & $\begin{array}{l}f_{i} \\
(9)\end{array}$ & $\begin{array}{l}\text { Rang } \\
\text { (10) }\end{array}$ & $\begin{array}{l}X_{f}^{2} \\
(I I)\end{array}$ & $\begin{array}{l}X_{f-i}^{2} \\
(12)\end{array}$ & $\begin{array}{l}C_{i} \\
(13)\end{array}$ & $\begin{array}{l}f_{i} \\
(14)\end{array}$ & $\begin{array}{l}\text { Rang } \\
\text { (15) }\end{array}$ \\
\hline Prestataire a parlé de la PF ? & 273,66 & 256,42 & 6,30 & 7,04 & 4 & 756,24 & 750,83 & 0,71 & 1,00 & 7 & 382,94 & 373,10 & 2,57 & 4,11 & 8 \\
\hline Ethnie & 273,66 & 267,92 & 2,10 & 2,34 & 10 & 756,24 & 753,37 & 0,38 & 0,53 & 10 & 382,94 & 379,30 & 0,95 & $\mathrm{I}, 5 \mathrm{I}$ & 10 \\
\hline Religion & 273,66 & 260,07 & 5,00 & 5,55 & 6 & 756,24 & 751,36 & 0,64 & 0,91 & 9 & 382,94 & 363,61 & 5,05 & 8,04 & 5 \\
\hline Degré de modernité & 273,66 & 267,08 & 2,40 & 2,68 & 9 & 756,24 & 748,22 & 1,06 & 1,49 & 4 & 382,94 & 373,24 & 2,53 & 4,04 & 9 \\
\hline Occupation du conjoint & 273,66 & 263,84 & 3,60 & 4,01 & 7 & 756,24 & 748,47 & 1,03 & $\mathrm{I}, 44$ & 6 & 382,94 & 369,32 & 3,56 & 5,67 & 6 \\
\hline
\end{tabular}

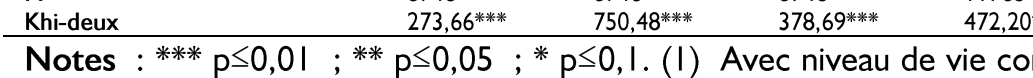

concept ; NU : Variable non utilisée ; ND : Variable non disponible.

Tableau 4: Hiérarchisation des variables selon leur contribution à l'explication des BNS en PF en 1995-96

http://aps.journals.ac.za

4710 


\begin{tabular}{|c|c|c|c|c|c|c|c|c|c|c|c|c|c|c|c|}
\hline Age $\mathrm{I}^{\mathrm{er}}$ mariage & 273,66 & 257,81 & 5,79 & 6,47 & 5 & 756,24 & 748,35 & 1,04 & 1,47 & 5 & 382,94 & 359,81 & 6,04 & 9,62 & 4 \\
\hline Proportion enfants décédés & 273,66 & 195,86 & 28,43 & 31,77 & I & 756,24 & 729,37 & 3,55 & 4,99 & 2 & 382,94 & 328,24 & 14,28 & 22,76 & 2 \\
\hline Nombre enfants survivants & 273,66 & 217,62 & 20,48 & 22,89 & 2 & 756,24 & 308,58 & 59,20 & 83,12 & I & 382,94 & $35 ।, 55$ & 8,20 & 13,06 & 3 \\
\hline Discussion au sein du couple & 273,66 & 266,48 & 2,62 & 2,93 & 8 & 756,24 & 751,10 & 0,68 & 0,95 & 8 & 382,94 & 369,49 & 3,54 & 5,63 & 7 \\
\hline Attitudes du couple & 273,66 & 230,63 & 12,80 & 14,30 & 3 & 756,24 & 734,19 & 2,91 & 4,10 & 3 & 382,94 & 321,50 & 16,04 & 25,56 & 1 \\
\hline
\end{tabular}

Notes: $\mathrm{X}_{\mathrm{f}}^{2}:$ Khi-deux du modèle saturé $; \mathrm{X}_{\mathrm{f}-\mathrm{i}}^{2}:$ Khi-deux sans la variable $\mathrm{x}_{\mathrm{i}} ; \mathrm{C}_{\mathrm{i}}=\left(\mathrm{X}_{\mathrm{f}^{-}}^{2} \mathrm{X}_{\mathrm{f}-\mathrm{i}}^{2} / \mathrm{X}_{\mathrm{f}}^{2} ; \mathrm{fi}=\frac{C i}{\sum_{i}^{k} C i}\right.$

Tableau 5: Hiérarchisation des variables selon leur contribution à l'explication des BNS en PF en 2006 (modèles contenant l'occupation du conjoint)

\begin{tabular}{|c|c|c|c|c|c|c|c|c|c|c|c|c|c|c|c|}
\hline \multirow[t]{2}{*}{ Variables } & \multicolumn{5}{|l|}{ Espacer } & \multicolumn{5}{|l|}{ Limiter } & \multicolumn{5}{|l|}{ Total } \\
\hline & $\begin{array}{l}X_{f}^{2} \\
(I)\end{array}$ & $\begin{array}{l}X_{f-i}^{2} \\
(2)\end{array}$ & $\begin{array}{l}C_{i} \\
\text { (3) }\end{array}$ & $\begin{array}{l}f_{i} \\
(4)\end{array}$ & $\begin{array}{l}\text { Rang } \\
\text { (5) }\end{array}$ & $\begin{array}{l}X_{f}^{2} \\
(6)\end{array}$ & $\begin{array}{l}X_{f-i}^{2} \\
(7)\end{array}$ & $\begin{array}{l}C_{i} \\
(8)\end{array}$ & $\begin{array}{l}f_{i} \\
(9)\end{array}$ & $\begin{array}{l}\text { Rang } \\
\text { (10) }\end{array}$ & $\begin{array}{l}\mathrm{X}_{\mathrm{f}}^{2} \\
\text { (II) }\end{array}$ & $\begin{array}{l}X_{f-i}^{2} \\
(I 2)\end{array}$ & $\begin{array}{l}C_{i} \\
(13)\end{array}$ & $\begin{array}{l}f_{i} \\
(14)\end{array}$ & $\begin{array}{l}\text { Rang } \\
\text { (15) }\end{array}$ \\
\hline Reçu visites des ASC ? & 473,65 & 431,06 & 8,99 & 9,91 & 3 & $148 \mid, 59$ & 1479,78 & 0,12 & 0,14 & il & 538,80 & 502,55 & 6,73 & 7,29 & 3 \\
\hline Prestataire a parlé de la PF ? & 473,65 & 433,64 & 8,45 & 9,31 & 4 & $148 \mid, 59$ & 1471,59 & 0,67 & 0,79 & 7 & 538,80 & 504,80 & 6,31 & 6,84 & 4 \\
\hline Ethnie & 473,65 & 463,29 & 2,19 & 2,41 & 9 & $148 \mid, 59$ & $|457,2|$ & 1,65 & 1,92 & 6 & 538,80 & 518,84 & 3,70 & 4,02 & 6 \\
\hline Religion & 473,65 & 470,11 & 0,75 & 0,82 & II & 1481,59 & 1471,97 & 0,65 & 0,76 & 8 & 538,80 & 534,08 & 0,88 & 0,95 & 10 \\
\hline Degré de modernité & 473,65 & 458,54 & 3,19 & 3,52 & 8 & $148 \mid, 59$ & 1475,48 & 0,41 & 0,48 & 9 & 538,80 & 522,41 & 3,04 & 3,30 & 9 \\
\hline Occupation du conjoint & 473,65 & 448,87 & 5,23 & 5,77 & 6 & $148 \mid, 59$ & 1442,77 & 2,62 & 3,07 & 4 & 538,80 & 507,67 & 5,78 & 6,26 & 5 \\
\hline Age $1^{\mathrm{er}}$ mariage & 473,65 & 465,52 & 1,72 & 1,89 & 10 & 1481,59 & 1457,12 & 1,65 & 1,93 & 5 & 538,80 & 530,10 & 1,61 & $\mathrm{I}, 75$ & 8 \\
\hline Proportion enfants décédés & 473,65 & 445,38 & 5,97 & 6,58 & 5 & 1481,59 & 1399,49 & 5,54 & 6,48 & 2 & 538,80 & 504,62 & 6,34 & 6,88 & 4 \\
\hline Nombre enfants survivants & 473,65 & 352,60 & 25,56 & 28,18 & I & $148 \mid, 59$ & 476,89 & 67,81 & 79,33 & I & 538,80 & 403,65 & 25,08 & 27,20 & 2 \\
\hline Discussion au sein du couple & 473,65 & 457,24 & 3,46 & 3,82 & 7 & 1481,59 & 1478,84 & 0,19 & 0,22 & 10 & 538,80 & 526,45 & 2,29 & 2,49 & 7 \\
\hline Attitudes du couple & 473,65 & 354,28 & 25,20 & 27,79 & 2 & $148 \mid, 59$ & $14 \mid 9,82$ & 4,17 & 4,88 & 3 & 538,80 & 374,68 & 30,46 & 33,02 & i \\
\hline
\end{tabular}

Notes : idem tableau 4.

Tableau 6: Hiérarchisation des variables selon leur contribution à l'explication des BNS en PF en 2006 (modèles contenant le niveau de vie)

\begin{tabular}{|c|c|c|c|c|c|c|c|c|c|c|c|c|c|c|c|}
\hline \multirow[t]{2}{*}{ Variables } & \multicolumn{5}{|l|}{ Espacer } & \multicolumn{5}{|l|}{ Limiter } & \multicolumn{5}{|l|}{ Total } \\
\hline & $\begin{array}{l}X_{f}^{2} \\
(I)\end{array}$ & $\begin{array}{l}X_{f-i}^{2} \\
(2)\end{array}$ & $\begin{array}{l}C_{i} \\
\text { (3) }\end{array}$ & $\begin{array}{l}f_{i} \\
(4)\end{array}$ & $\begin{array}{l}\text { Rang } \\
\text { (5) }\end{array}$ & $\begin{array}{l}X_{f}^{2} \\
(6)\end{array}$ & $\begin{array}{l}X_{f-i}^{2} \\
(7)\end{array}$ & $\begin{array}{l}C_{i} \\
(8)\end{array}$ & $\begin{array}{l}f_{i} \\
(9)\end{array}$ & $\begin{array}{l}\text { Rang } \\
(10)\end{array}$ & $\begin{array}{l}X_{f}^{2} \\
(I I)\end{array}$ & $\begin{array}{l}X_{f-i}^{2} \\
(I 2)\end{array}$ & $\begin{array}{l}\mathrm{C}_{\mathrm{i}} \\
(13)\end{array}$ & $\begin{array}{l}f_{i} \\
(14)\end{array}$ & $\begin{array}{l}\text { Rang } \\
\text { (15) }\end{array}$ \\
\hline Reçu visites des ASC ? & 472,20 & 425,77 & 9,83 & 10,09 & 3 & 1463,18 & 459,40 & 0,26 & 0,30 & 10 & 517,11 & 481,20 & 6,94 & 7,52 & 3 \\
\hline Prestataire a parlé de la PF ? & 472,20 & 427,82 & 9,40 & 9,65 & 4 & 1463,18 & 1449,42 & 0,94 & 1,10 & 7 & 517,11 & 483,46 & 6,51 & 7,05 & 4 \\
\hline Ethnie & 472,20 & 457,23 & 3,17 & 3,26 & 9 & 1463,18 & $|435,5|$ & $\mathrm{I}, 89$ & 2,20 & 4 & 517,11 & 493,77 & 4,51 & 4,89 & 7 \\
\hline Religion & 472,20 & 469,29 & 0,62 & 0,63 & II & 1463,18 & 1452,89 & 0,70 & 0,82 & 8 & 517,11 & 513,57 & 0,68 & 0,74 & II \\
\hline Degré de modernité & 472,20 & 442,78 & 6,23 & 6,40 & 5 & 1463,18 & $|459,2|$ & 0,27 & 0,32 & 9 & $5 \mid 7,11$ & 492,51 & 4,76 & 5,15 & 6 \\
\hline Niveau de vie & 472,20 & 448,87 & 4,94 & 5,07 & 7 & 1463,18 & 1442,77 & 1,39 & 1,62 & 6 & 517,11 & 507,67 & 1,82 & 1,98 & 9 \\
\hline Age $1^{\mathrm{er}}$ mariage & 472,20 & 462,95 & 1,96 & 2,01 & 10 & 1463,18 & $144 \mid, 90$ & 1,45 & 1,70 & 5 & 517,11 & 508,39 & 1,69 & 1,83 & 10 \\
\hline Proportion enfants décédés & 472,20 & 446,84 & 5,37 & 5,51 & 6 & 1463,18 & 1384,57 & 5,37 & 6,26 & 2 & 517,11 & 485,62 & 6,09 & 6,60 & 5 \\
\hline Nombre enfants survivants & 472,20 & 344,26 & 27,09 & 27,81 & $\mathrm{I}$ & 1463,18 & $45 I, 40$ & 69,15 & 80,60 & I & 517,11 & 385,32 & 25,49 & 27,61 & 2 \\
\hline Discussion au sein du couple & 472,20 & 453,25 & 4,01 & 4,12 & 8 & 1463,18 & 1460,48 & 0,18 & 0,22 & II & 517,11 & 504,15 & 2,51 & 2,71 & 8 \\
\hline Attitudes du couple & 472,20 & 355,15 & 24,79 & 25,45 & 2 & 1463,18 & 1402,20 & 4,17 & 4,86 & 3 & 517,11 & 355,15 & 31,32 & 33,92 & $\mathrm{I}$ \\
\hline
\end{tabular}

Notes : ibid.

Tableau 7: Hiérarchisation des variables selon leur contribution à l'explication des BNS en PF en 20 I2-I3

\begin{tabular}{|c|c|c|c|c|c|c|c|c|c|c|c|c|c|c|c|}
\hline \multirow[t]{2}{*}{ Variables } & \multicolumn{5}{|l|}{ Espacer } & \multicolumn{5}{|l|}{ Limiter } & \multicolumn{5}{|l|}{ Total } \\
\hline & $\begin{array}{l}X_{f}^{2} \\
(I)\end{array}$ & $\begin{array}{l}X_{f-i}^{2} \\
(2)\end{array}$ & $\begin{array}{l}C_{i} \\
\text { (3) }\end{array}$ & $\begin{array}{l}f_{i} \\
(4)\end{array}$ & $\begin{array}{l}\text { Rang } \\
\text { (5) }\end{array}$ & $\begin{array}{l}X_{f}^{2} \\
(6)\end{array}$ & $\begin{array}{l}X_{f-i}^{2} \\
(7)\end{array}$ & $\begin{array}{l}C_{i} \\
(8)\end{array}$ & $\begin{array}{l}f_{i} \\
(9)\end{array}$ & $\begin{array}{l}\text { Rang } \\
(10)\end{array}$ & $\begin{array}{l}X_{f}^{2} \\
\text { (II) }\end{array}$ & $\begin{array}{l}X_{f-i}^{2} \\
(12)\end{array}$ & $\begin{array}{l}C_{i} \\
(13)\end{array}$ & $\begin{array}{l}f_{i} \\
(14)\end{array}$ & $\begin{array}{l}\text { Rang } \\
\text { (15) }\end{array}$ \\
\hline Reçu visites des ASC ? & 122,06 & 122,05 & 0,01 & 0,01 & 7 & 656,32 & 650,68 & 0,86 & $\mathrm{I}, 09$ & 5 & 259,78 & 257,90 & 0,72 & $\mathrm{I}, 10$ & 9 \\
\hline Prestataire a parlé de la PF ? & 122,06 & 113,32 & 7,16 & 8,84 & 3 & 656,32 & 647,88 & 1,28 & 1,62 & 4 & 259,78 & 243,89 & 6,12 & 9,26 & 3 \\
\hline Ethnie & 122,06 & 119,44 & 2,15 & 2,65 & 6 & 656,32 & 644,92 & $\mathrm{I}, 74$ & 2,20 & 3 & 259,78 & 253,90 & 2,26 & 3,43 & 6 \\
\hline
\end{tabular}


African Population Studies Vol.33, No.I, Feb. 2019

\begin{tabular}{|c|c|c|c|c|c|c|c|c|c|c|c|c|c|c|c|}
\hline Religion & 122,06 & 115,86 & 5,08 & 6,27 & 4 & 656,32 & 653,24 & 0,47 & 0,59 & 8 & 259,78 & 254,92 & $\mathrm{I}, 87$ & 2,83 & 7 \\
\hline Degré de modernité & 122,06 & 108,50 & $\mathrm{II}, \mathrm{II}$ & $|3,7|$ & 2 & 656,32 & 655,20 & 0,17 & 0,22 & 9 & 259,78 & 248,18 & 4,46 & 6,76 & 4 \\
\hline Niveau de vie & 122,06 & 108,50 & 11,11 & $|3,7|$ & 2 & 656,32 & 651,94 & 0,67 & 0,84 & 7 & 259,78 & 251,79 & 3,08 & 4,66 & 5 \\
\hline Age $\mathrm{I}^{\mathrm{er}}$ mariage & 122,06 & 118,03 & 3,30 & 4,07 & 5 & 656,32 & 651,36 & 0,76 & 0,96 & 6 & 259,78 & 257,39 & 0,92 & 1,39 & 8 \\
\hline Proportion enfants décédés & 122,06 & 96,82 & 20,68 & 25,52 & I & 656,32 & 636,67 & 2,99 & 3,78 & 2 & 259,78 & 241,89 & 6,89 & 10,43 & 2 \\
\hline Nombre enfants survivants & 122,06 & 97,12 & 20,43 & 25,22 & 1 & 656,32 & 195,68 & 70,18 & 88,70 & 1 & 259,78 & 156,57 & 39,73 & 60,15 & 1 \\
\hline
\end{tabular}


Concernant le risque de BNS pour limiter les naissances, aux trois périodes, seulement le nombre d'enfants survivants explique plus de $60 \%$ de sa variation (tableau 4 à 7 : colonne 10). Quant au total de BNS en PF, quelle que soit la période, les variables qui contribuent le plus à sa variation sont similaires à celles mises en exergue dans le cas des BNS pour espacer les naissances (tableau 4 à 7 : colonne I5).

\section{Variation du risque de $\mathrm{BNS}$ en $\mathrm{PF}$}

En 2012-13, l'une des deux variables relatives à l'offre, à savoir le fait d'avoir reçu l'information sur la PF dans le centre santé, est associée négativement à toutes les trois variables dépendantes $(O R=0,69$ dans le cas de l'espacement ou de la limitation des naissances et 0,65 dans le cas du total des BNS) (revoir tableau 3).

S'agissant des variables relatives à la demande, en 1995-96, les résultats obtenus confirment l'hypothèse selon laquelle les femmes musulmanes ont plus de risque de $B N S$ en PF que les animistes $(O R=0,60)$. En 20I2-13, c'est par rapport aux chrétiennes $(O R=0,74)$ que les premières se démarquent dans le même sens. En revanche, en 2006, aucune différence significative n'est observée entre les musulmanes et l'une ou l'autre catégorie susmentionnée de la religion. Ce qui témoigne du fait que les premières seraient plus attachées aux valeurs traditionnelles de PF que les autres et converge dans le même sens que les résultats issus des travaux d'autres chercheurs notamment Akoto et Kamdem (200l) sur le Cameroun, Kenya et Ethiopie, Evina (2005) sur le premier pays et Congo (2005) sur le Burkina Faso. En revanche, il contredit ceux de Kourouma (20II) sur la Guinée.

Dans le cas de l'autre indicateur du milieu socioculturel traditionnel, à savoir l'ethnie, l'hypothèse selon laquelle le risque de BNS en PF pour l'espacement des naissances ne varie pas selon ses modalités n'a été confirmée qu'en 2012-13. Dans le cas des deux autres variables dépendantes, de 2006 à 2012-13, on a constaté que les différences ethniques n'ont pas disparu, mais elles se sont considérablement réduites.

Contre toute attente, quelle que soit la période, toutes choses égales par ailleurs, le degré de modernité culturelle est positivement associé aux variables dépendantes étudiées, à l'exception des BNS en PF pour limiter les naissances en 2006 et $2012-13$, où son influence est non significative. Les relations entre l'occupation du conjoint ou le niveau de vie et les BNS en PF vont partiellement dans le sens attendu. En effet, en 1995-96 et 2006, les femmes dont les conjoints sont cadres/employés se démarquent bien de celles d'agriculteurs par un faible risque de BNS en PF dans le cas de l'espacement des naissances $(O R=0,66$ et 0,68$)$ ou du total des $B N S$ en PF (OR=0,68 et $0,7 \mathrm{I})$ alors que dans le cas de la limitation des naissances la différence entre les deux groupes s'est avérée non significative. Pour le niveau de vie, en 2006 et 2012-I3, par rapport au groupe de référence, au moins une des modalités de niveau de vie supérieur est négativement associée au risque de BNS en PF dans le cas de l'espacement des naissances et du total des BNS. Lorsqu'on s'intéresse aux BNS en PF pour limiter les naissances, l'influence du niveau de vie s'est avérée soit non significative soit positive.

S'agissant des variables sociodémographiques considérées, dans le cas de l'âge à la première union, c'est seulement en 1995-96 que l'hypothèse selon laquelle le risque de BNS en PF est plus faible chez les femmes ayant tardivement contracté leurs premières unions que chez celles qui les ont contractées avant I 5 ans est confirmée; en 2006, elle est partiellement confirmée; en 20I2-13, elle est infirmée. En effet, à cette dernière période, contre toute attente, nous avons constaté que l'influence de l'âge à la première union est non significative dans les cas des BNS pour espacer les naissances et du total des BNS et que le risque de BNS pour limiter les naissances est plus élevé chez les femmes ayant très tardivement contracté leurs premières unions que chez d'autres. Pour la proportion d'enfants décédés, quelle que soit la période, conformément à l'attente, elle est négativement associée au risque de BNS en PF dans le cas de l'espacement des naissances et du total des BNS. En revanche, elle est positivement associée au risque de BNS en PF pour limiter les naissances. Quant au nombre d'enfants survivants, conformément à l'attente, il est positivement lié au risque de BNS en PF dans les cas de la limitation des naissances et du total des BNS. En revanche, il est négativement lié au risque de BNS en PF pour l'espacement des naissances.

Dans l'ensemble des indicateurs des rapports de genre, en 1995-96 et 2006, la discussion au sein du couple est partiellement associée dans le sens attendu aux BNS pour espacer les naissances et au total des BNS. En revanche, aux deux périodes, elle n'est pas associée aux BNS pour limiter les naissances. Contre toute attente, aux deux périodes, les attitudes favorables à la PF au sein du couple augmentent plutôt le risque de BNS en PF.

Pour expliquer la plupart des relations inattendues observées dans le milieu étudié, nous avons procédé à la triangulation des données, en confrontant ces 
relations aux informations qualitatives sur les éléments des contextes sanitaire et politique de PF (non présentées ici). En 1995-96 et 2006, les risques positifs de BNS en PF observés dans les couches sociales favorables à la $\mathrm{PF}$ résultent de l'insuffisance à ces périodes de l'offre de PF. En 20/2-13, ils s'expliquent par le fait que l'amélioration de l'offre de PF, qui a eu lieu dans ce milieu après 2006, ne permet pas encore aux individus de ces couches sociales (particulièrement ceux de niveau élevé de modernité culturelle, de niveau de vie très élevé et ayant des attitudes favorables vis-à-vis de la PF) de satisfaire une bonne partie de leurs besoins en PF, particulièrement dans le cas de la limitation des naissances.

\section{Conclusions et recommandations}

Les résultats issus des analyses des données des EDS réalisées au Mali en 1995-96, 2006 et 2012-13 ont permis d'atteindre les objectifs spécifiques de l'étude. En effet, il en est ressorti qu'en 1995-96 les facteurs du risque de BNS en PF appartiennent seulement à l'ensemble des variables indépendantes relatives à la demande, et on les retrouve dans tous les types de variables considérées, alors qu'en 2006 et 2012-13, ils appartiennent aussi à l'ensemble des variables indépendantes relatives à l'offre. A la première période, trois variables, à savoir la proportion d'enfants décédés, le nombre d'enfants survivants et les attitudes des couples vis-à-vis de la PF, contribuent le plus à l'explication de la variance du risque de BNS en PF pour espacer les naissances ; en 2006 et 2012-13, on retrouve en plus les visites à domiciles de ASC ou la réception des informations sur la PF dans les centres de santé pendant les consultations médicales. Quant aux BNS pour limiter les naissances, quelle que soit la période, le nombre d'enfants survivants explique seul presque la totalité de sa variance. De même, concernant le total des BNS en PF, ses déterminants les plus importants sont similaires à ceux du risque de BNS en PF pour espacer les naissances.

Par ailleurs, les résultats de l'étude ont confirmé totalement ou partiellement la plupart des hypothèses de travail relatives aux effets des différentes variables indépendantes sur le risque de BNS en PF, particulièrement dans les cas de l'espacement des naissances et du total des BNS.

La contribution de l'étude dans l'amélioration des connaissances sur les facteurs explicatifs des BNS en PF est grande. La revue de la littérature avait révélé qu'il y a très peu de travaux scientifiques portant sur le Mali dans lesquels les facteurs de la PF et/ou du risque de BNS en PF ont été mis en exergue. Une seule étude, notamment celle de Mariko et al. (2009) a porté sur ce dernier sujet, mais les données qu'ils ont utilisées sont anciennes et en plus leur recherche des facteurs n'a pas été précédée d'une réflexion théorique. En s'orientant vers une approche globale d'explication, intégrant les variables de l'offre et celles de la demande, et vers une approche diachronique d'analyse, dans laquelle les données anciennes et les plus récentes ont été utilisées, cette étude a permis de mieux expliquer les variations des BNS en PF au Mali et de montrer que l'amélioration de l'offre de PF s'est, dans une certaine mesure, répercutée positivement dans les pratiques préventives des populations dans le domaine de la santé reproductive. Par ailleurs, comme les analyses effectuées ont, entre autres choses, permis d'hiérarchiser les facteurs, l'étude contient les informations auxquelles le Mali devrait se référer pour identifier les actions à entreprendre en priorité pour réduire le niveau de BNS en PF.

Les résultats de l'étude suggèrent un certain nombre de mesures prioritaires profitables pour la PF au Mali. Sur le plan politique, il est important d'améliorer l'offre de PF, particulièrement de permettre aux femmes d'être pour la plupart suffisamment informées sur la PF, lors de leur fréquentation de centres de santé, pour les visites prénatales ou d'autres problèmes de santé reproductive. Sur le même plan, il est aussi important de rendre accessibles toutes les méthodes modernes de contraception d'espacement et de limitation des naissances afin que les couples et les individus motivés à les utiliser $y$ accèdent facilement. Le renforcement des stratégies de réduction de la mortalité des enfants est une autre action importante à entreprendre puisque nous avons constaté que le décès d'enfants est négativement associé aux motivations pour l'utilisation de la contraception d'espacement des naissances. En même temps que les actions précédentes, les conditions de vie des familles devraient être améliorées, en permettant, par exemple, aux femmes pauvres de réaliser des activités génératrices des revenus.

Par ailleurs, compte tenu du fait que cette étude a révélé, à partir des données plus récentes, que la religion influence significativement dans le sens attendu les BNS en PF, le Gouvernement du Mali et ses partenaires devraient s'appuyer sur les responsables religieux, pour améliorer les comportements contraceptifs des femmes. La population malienne étant presque totalement d'obédience musulmane, ils devraient particulièrement s'appuyer sur les Imams puisqu'ils ont une grande autorité morale dans cette population. On briserait suffisamment les résistances en matière de planification familiale en agissant de cette façon. En effet, si l'on en croit notamment Bijlmakers et al. (2009), au Mali, à cause des 
pressions des leaders religieux et traditionnels, le nouveau code des personnes et de la famille, dans lequel les lois favorables à l'indépendance des Bec femmes ont été incluses, avait été adopté plusieurs années après sa première présentation en 2009 aux parlementaires et, ce, après que les lois Bella, J N 1998 : Les politiques de population dans deux susmentionnées y soient retirées ou amandées.

Enfin, dans cette étude, l'approche explicative utilisée est celle intégrant les variables de l'offre et celles de la demande, mais dans l'ensemble des B variables indépendantes utilisées, nous n'avons inclus que deux indicateurs de l'offre puisque très peu d'informations y relatives ont été collectées pendant les EDS I à $V$ réalisées au Mali. $A$ cet effet, une des $B$ pistes pour les recherches futures serait d'inclure le questionnaire communautaire (contenant assez d'informations sur l'offre de PF) dans les outils de collecte des données pendant les EDS et de recourir aux approches multi-niveaux d'analyse des données, afin d'évaluer les effets des variables indépendantes $B$ sur les BNS en PF en les distinguant bien selon leur niveau d'intervention: communautaire, familial et individuel. Ce qui permettra aussi d'évaluer l'apport de chaque groupe des variables à l'explication du Ca risque de BNS en PF.

\section{Références bibliographiques}

Agwanda, A, Awes, A et Muia, R 2015, «Family Planning», in Kenya National Bureau of Statistics, Ministry of Health, National Aids Control Council et al. (eds), National Kenya Demographic and Health C Survey, décembre 2015, pp. 89-1 I0.

Ahovey, E C 2002, «Besoins Non Satisfaits en Planification Familiale au sein du couple: Cochrane, S H et Guilkey, D K 1995, "The effects of caractéristiques sociodémographiques et cadre de vie au Bénin», Interregional Seminar on Reproductive Health, unmet need and poverty: issues of success and and quality of services, Bangkok, Thailand, 25-29 novembre 2002, CICRED, 28p. Cor [http://www.cicred.org/Eng/Seminars/Details/Seminar s/Bangkok2002/00 IBangkokAhovey.pdf] (consulté le II février 2017).

Akoto, E M, Tambashe, O, Amouzou, A et Djangone, A C $M R$ 2002, "Besoins non-satisfaits en planification familiale et transition contraceptive au Burkina Faso, au Cameroun et en Côte d'lvoire ", Etude de la Population Africaine, vol. 17, n'I ; pp. 19-45.

Akoto, E M et Kandem, H 2000, "Étude comparative Cr des déterminants de la pratique contraceptive moderne en Afrique ", in Gendreau F. (eds), La transition démographique des pays du Sud, Éditions Estem, pp. 27I-285.

Arends-Kuenning, M et Kessy, F L 2007, «The Impact of Demand Factors, Quality of Care and Access to
Facilities on Contraceptive Use in Tanzania», Journal of Biosocial Science, volume 39, $n^{\circ}$ I: I 26.

Becker, G S 1960, «New Economic Approaches to Fertility", The Journal of Political Economy, vol. 8I, $n^{\circ}$ 2, part 2: (Mar. - Apr., 1973); pp. 279-288.

pays en développement: le Cameroun et la Thaillande, Les cahiers de I'IFORD, $n^{\circ}$ I7, YaoundéCameroun, 156p.

Belohlav, K et Karra, M 2013, "La prise des décisions des ménages et l'utilisation de la contraception en Zambie ", Note de recherche, Population Reference Bureau, novembre 2013, 5p.

ijlmakers, L, Carr-Hill, R, Doumbia, S, Kanté, N, Diarra, $\mathrm{K}$ et al. 2012 : Pratique contraceptive et la contribution des activités de planification familiale au Mali. Une étude dans le cadre de la coopération malienne - néerlandaise, Consortium ETC, Ecorys, KIT, CDR et ETC Crystal, Bamako, août 20I2, I26p.

lanc, A K, Curtis, S L et Croft, T N, 2002, «Monitoring Contraceptive Continuation: Links to Fertility Outcomes and Quality of Care», Studies in Family Planning, 3 (2); pp. I27-40.

Caldwell, J C 1976, "Toward a Restatement of Demographic Transition Theory», Population and Development Review, vol.2, N4; pp.32I-366.

Caldwell, J C 1982, "Theory of Fertility Decline, Population and Social Structure: Advances in Historical Demography», Academic Press; pp. 458493

Caldwell, J C 1983, «Direct economic cost and benefits of children», Determinants of Fertility in Developing Countries, vol.23, n 4 ; pp. 803-8I2.

fertility intentions and access to services on contraceptive use in Tunisia», Economic Development and cultural change, volume $43, n^{\circ} 4$; pp. 779-804.

Congo, Z 2005, Les facteurs de la contraception au Burkina Faso, Groupe International des Partenaires Population et Santé (GRIPPS), la planification familiale en Afrique, Document d'analyse $n^{\circ} 5,53 p$.

CPS/SSDSPF, INSTAT/MPATP, INFO-STAT et ICF International 2014, "Planification Familiale. Enquête Démographique et de Santé au Mali 2012-2013 ", Rockville, Maryland, USA : CPS, INSTAT, INFOSTAT et ICF International. ; pp. 85-I 04.

Creel, L C, Sass, J V et Yinger, N V 2002, «Overview of Quality of Care in Reproductive Health: Definitions and Measurements of Quality», New Perspectives on Quality of Care, volume I, pp. I-8.

Dembele, E et Keita, O 2007, «Planification Familiale», Cellule de Planification et de Statistique du Ministère de la Santé (CPS/MS), Direction Nationale de la 
Statistique et de l'Informatique du Ministère de Koenig, M, Hossain, M et Whittaker, M 1997, The l'Économie, de l'Industrie et du Commerce Influence of Quality of Care Upon contraceptive Use (DNSI/MEIC) et Macro International Inc. (eds), in Rural Bangladesh», Studies in Family Planning, Enquête Démographique et de Santé du Mali 2006, volume 28, n²; pp. 278-89.

Calverton, Maryland, USA: CPS/DNSI et Macro Kourouma, N 20II, Relations entre le niveau de vie, la International Inc. ; pp. 63-80.

Easterlin, R A 1975. "An Economic Framework for Fertility Analysis», Studies in Family Planning, volume 6, n³; pp.54-63.

Ela, J M 1995, "Fécondité, structures sociales et juin 20II, 305p.

fonctions dynamiques de l'imaginaire en Afrique noire Locoh, T et Makdessi, Y 2000, «Politique de population ", in Hubert Gérard et Victor Piché (eds), Sociologie des populations, Éd. Presses de l'Université de Montréal, AUPELF/UREF ; pp.II7-I37.

Evina, A 2005, Les facteurs de la contraception au Cameroun, Groupe International des Partenaires Population et Santé (GRIPPS), la planification familiale Mariko, S, Ayad, M, Hong, R et al. 2009, Pratique en Afrique, Document d'analyse $n^{\circ} 6,47 p$.

Fomekong, F et Tchekanda, E 20I2, "Planification Familiale ", in Institut National de la Statistique (INS) et ICF. International. (eds), Enquête Démographique et de Santé et à Indicateurs Multiples du Cameroun 20II, Calverton, Maryland, USA : INS et ICF International. ; Pp. I0I-II8.

et baisse de la fécondité en Afrique sub-saharienne ", in Vimard, $P$ et Zanou, B (éds), Politiques démographiques et transition de la fécondité en Afrique, L'Harmattan, Paris (France), Collection Populations ; pp. 263-296.

contraceptive et importance des besoins non satisfaits en matière de planification familiale au Mali, de 1995 à 2006. Analyses approfondies des Enquêtes Démographiques et de Santé au Mali, 1995-1996, 200 I et 2006, Rapports d'analyses approfondies des EDS No. 69, Calverton, Maryland, USA : CPS/DNSI et ICF Macro., septembre 2009, 43p.

Freedman, R, Coombs, L C et Chang, M 1972, «Trends Measurement, Learning and Evaluation Project (MLE) in family size preferences and practice of family Planning: Taiwan, 1965-1970 », Studies in Family Planning, volume 3, n I 2; pp.28I-296.

Gérard, H 1995, «Pour une sociologie de la population», in H. Gérard et V. Piché (eds), La Sociologie des populations, AUPELF/UREF, PUM; pp.32I-37.

2012 : L'efficacité des interventions de planification familiale, Rapport de recherche I-20I2, 8p.

Mroz, T, Bollen, K A, Speizer, I S et Mancine, D J 20I5, "Quality, accessibility, and contraceptive use in rural Tanzania», In Demography, February 1999, volume 36, issue I; pp. 23-40.

Ghana Statistical Service (GSS), Ghana Health Service Ndiaye, C T et Diop P M 20I2, «Planification Familiale », (GHS), and ICF International. 20I5. Ghana Demographic and Health Survey 2014, Rockville, Maryland, USA: GSS, GHS, and ICF International; pp. 79-98.

Gilda S., Lori S., Rubina H. (2016), Unmet need for contraception in developing countries : examining women's reasons for not using a method, New York RamaRao, S, Lacuesta, M, Costello, M, Pangolibay, B et : Guttmacher Institute 2016, 93p.

Gregory, J W et Piché, V 1985, «Mode de production et régime démographique », Canadian Journal of African studies, $\mathrm{n}^{\circ} 19$; pp.73-77.

Gribble, J et Voss, M L 2009, Planification Familiale et Bien-être économique : Le Bangladesh fait ses preuves, Population Reference Bureau, 4p.

Hassane, R, Nomaou, A et Seydou, M 2013, " in Agence Nationale de la Statistique et de la Démographie (ANSD) et ICF International. (eds), Enquête Démographique et de Santé à Indicateurs Multiples au Sénégal (EDS-MICS) 2010-20II, Calverton, Maryland, USA: ANSD et ICF International ; pp. 97-I I4.

Jones, H 2003, "The Link between Quality of Care and Contraceptive Use», International Family Planning Perspectives, volume 29, n²; pp.76-83.

Sanni, A 20I I, "Niveaux et tendances des Besoins Non Satisfaits en Planification Familiale : Facteurs Explicatifs et Changements dans le temps ", African Population Studies, volume 25, n² (Dec 201 I) ; pp. $38|-40|$.

Planification Familiale ", Institut National de la Sanogo, D, RamaRao, S, Jones, H, N'diaye, P, M'bow, B Statistique (INS) et ICF International (eds), Enquête Démographique et de Santé et à Indicateurs Multiples du Niger 2012, Calverton, Maryland, USA : INS et ICF International., septembre 2013 ; pp. 63-80.

et Diop, C B 2003, «Improving Quality of Care and Use of Contraceptives in Senegal», African Journal of Reproductive Health; pp. 57-73.

Kobiane J. F. (20I4), Progrès et défis de l'EPT en Afrique subsaharienne francophone : enseignements des enquêtes auprès des ménages, Cahiers de l'IFORD $n^{\circ} 32$, I58p.

Sanon, D et Tapsoba, E 2012, "Planification Familiale ", in Institut National de la Statistique et de la Démographie (INSD) et ICF International (20I2) : Enquête Démographique et de Santé et à Indicateurs 
Multiples du Burkina Faso 2010, Calverton, Maryland, USA : INSD et ICF International. ; pp. 68-84.

Speizer, I S et Bollen, K A 2000, «How well do perceptions of Family Planning Servive Quality corresponds to Objective measures? Evidence from Tanzania», Studies in Family Planning, volume $31, \mathrm{n}^{\circ} 2$, juin 2000; pp. 163-I 78.

Tiendrebeogo, A 2004, Statut de la femme, relations de genre et pratique contraceptive moderne : cas du Burkina Faso, Mémoire de DESSD, IFORD, Avril 2004, I19p.

Traoré, S M et Ballo, M B 2002, "Préférences en matière de fécondité ", in Cellule de Planification et de Statistique du Ministère de la Santé (CPS/MS), Direction Nationale de la Statistique et de I'Informatique (DNSI) et ORC Macro. (eds) : Enquête Démographique et de Santé au Mali 200I, Calverton, Maryland, USA : CPS/MS, DNSI et ORC Macro. ; pp. 95-106.

UNFPA et Path 2008, Besoins non satisfaits en planification familiale : Stratégies et approches factuelles, Outlook, numéro spécial, 25ème anniversaire, 8p.

Vimard, P et Fassassi, R 2007, "La demande d'enfants en Afrique subsaharienne ", in Ferry Benoît (eds) : L'Afrique face à ses défis démographiques : un avenir incertain, Paris (FRA) ; Nogent-sur- Marne (FRA) ; Paris, AFD/CEPED, (Éds.) Karthala, pp. I97-25I.

Wakam, J 2004 : De la pertinence des théories économistes de la fécondité en Afrique, Editions Academia, collection Thèses de sciences humaines I4, 527p.

Westoff, C F et Bankole, A 1995, «Unmet need: 19901994», DHS Comparative Studies n¹6, Calverton, Maryland, Macro International, 55p.

WHO 20ll: Preventing early pregnancy and poor reproductive outcomes, Geneva: WHO, 20II, 195p.

\section{Intérêts concurrents}

Nous déclarons n'avoir ni de relations personnelles ni financières qui auraient pu nous influencer de quelque manière que ce soit dans la rédaction de cet article.

\section{Contributions des auteurs}

Les deux auteurs ont identifié le problème et recherché la méthode adéquate. KS (chercheur à PSI Mali) s'est chargé de l'analyse des données des EDS qui ont été utilisées. RMJR (enseignant-chercheur à l'IFORD) a effectué la synthèse de la littérature, dégagé les différentes approches explicatives et l'approche théorique de l'étude et rédigé toutes les autres sections de l'étude. II s'est aussi chargé de travaux de mise en forme de l'article selon les normes de l'African Population Studies (APS).

\section{ANNEXES}

Annexe $n^{\circ} I$ : Création et validation de la variable degré de modernité culturelle

Le fait que nous ayons observé qu'il existe des liens entre les variables renseignant sur l'ouverture aux valeurs modernes signifie qu'ils ont tous tendance à apporter les mêmes types d'informations dans l'explication des BNS en PF. En effet, ils permettent tous de savoir à quel degré l'individu était ou n'était pas en contact avec les valeurs modernes, y compris celles relatives à la fécondité et PF. Le degré de modernité culturelle a été créé à l'Aide de l'Analyse en Composantes Principales (ACP). Etant donné que cette méthode d'analyse factorielle ne s'applique que sur des variables quantitatives, des variablesmodalités binaires ont été créées au niveau de chacune des variables susmentionnées et c'est sur elles que l'ACP a été appliqué. Pour créer un indicateur composite à partir de cette méthode, un seul facteur a été retenu et ceci était le critère de convergence. Pour chaque enquête, on a donc créé à l'aide de cette méthode le degré de modernité culturelle. Pour nous rassurer de sa cohérence interne, nous avons croisé la nouvelle variable avec chacune des anciennes variables et obtenu les résultats présentés au tableau $A$.I.

II ressort de ce tableau que la relation entre le degré de modernité culturelle et chacune des variables ayant participé à sa construction est conforme à celle qu'on attendait. Ce facteur a donc un degré de fiabilité élevé. Par exemple, dans le cas du milieu de résidence au moment de l'enquête, on constate que, quelle que soit la période, la proportion des femmes ciblées ayant un degré élevé de modernité culturelle est de $90-100 \%$ à Bamako, 65$76 \%$ dans les autres villes et $7-10 \%$ en milieu rural. Concernant le niveau d'instruction de la femme, quelle que soit la période, cette proportion vaut $95-$ $100 \%$ chez les femmes de niveau secondaire ou supérieur, $65-70 \%$ chez celles de niveau primaire et 13-17\% chez celles sans niveau d'instruction. Dans l'ensemble des variables de l'exposition aux médias, la lecture des journaux ou magazines et l'exposition à la télévision discriminent mieux le degré de modernité culturelle que l'exposition à la radio. En effet, quelle que soit la période, la proportion des femmes ayant un niveau élevé de modernité culturelle est proche de $100 \%$ chez celles ayant déclaré lire les journaux ou magazines contre 20-23 $\%$ chez celles ayant déclaré ne pas du tout les lire. En 1995-96, ces proportions sont de $68,2 \%$ et $10,7 \%$ 
African Population Studies Vol.33, No. I, Feb. 2019

respectivement chez celles ayant déclaré être exposées à la télévision et chez celles ayant déclaré ne pas du tout être exposées à ce média. En 2006, elles sont de $50,7 \%$ et $7 \%$ respectivement chez celles qui y sont exposées au moins une fois par semaine et chez celles qui n'y sont pas du tout exposées. En $2012-13$, elles sont de $62,7 \%$ et 4,7 $\%$. 
African Population Studies Vol.33, No. I, Feb. 2019

Tableau A. I: Degré de modernité culturelle selon les modalités de ses composantes (EDS, $1995-96$; 2006 et $2012-13$ )

\begin{tabular}{|c|c|c|c|c|c|c|c|c|c|c|c|c|}
\hline \multirow{2}{*}{$\begin{array}{l}\text { Indicateurs } \\
\text { Milieu enfance }\end{array}$} & \multicolumn{4}{|c|}{ EDS 1995-96 } & \multicolumn{4}{|c|}{ EDS 2006} & \multicolumn{4}{|c|}{ EDS $2012-13$} \\
\hline & $\mathrm{T}$ faible & Faible & Moyen & Elevé & $\mathrm{T}$ faible & Faible & Moyen & Elevé & $\begin{array}{l}\text { T faible } \\
\text { ND }\end{array}$ & $\begin{array}{l}\text { Faible } \\
\text { ND }\end{array}$ & $\begin{array}{l}\text { Moyen } \\
\text { ND }\end{array}$ & $\begin{array}{l}\text { Elevé } \\
\text { ND }\end{array}$ \\
\hline Bamako & - & - & 3,8 & 96,2 & - & - & 7,4 & 92,6 & & & & \\
\hline Autres villes & - & - & 12,6 & 87,4 & - & 3,0 & 24,7 & 72,3 & & & & \\
\hline Rural & 33,2 & 27,7 & 27,8 & 11,2 & 31,1 & 36,2 & 22,0 & 10,7 & & & & \\
\hline \multicolumn{13}{|l|}{ Milieu résidence } \\
\hline Bamako & - & - & 5,9 & 94,1 & - & - & 4,4 & 95,6 & - & - & 12,3 & 87,3 \\
\hline Autres villes & - & - & 30,4 & 69,6 & - & $\mathrm{I}, \mathrm{I}$ & 33,2 & 65,7 & - & 0,1 & 24,1 & 75,8 \\
\hline \multicolumn{12}{|l|}{ Instruction femme } & 10,0 \\
\hline Sans niveau & 32,1 & 26,8 & 24,6 & 16,5 & 28,6 & 33,4 & 21,5 & 16,4 & 30,8 & 29,7 & 26,5 & 13,0 \\
\hline Primaire & - & - & 34,2 & 65,8 & - & $4, I$ & 26,4 & 69,5 & - & 2,1 & 29,7 & 68,2 \\
\hline $\begin{array}{l}\text { Secondaire et }+ \\
\text { Instruction conjoint }\end{array}$ & - & - & 0,9 & 99,1 & - & - & 1,5 & 98,5 & - & - & 4,3 & 95,7 \\
\hline Sans niveau & 32,7 & 26,7 & 25,0 & 15,6 & 30,5 & 32,9 & 20,4 & 16,3 & 31,0 & 29,1 & 26,7 & 13,2 \\
\hline Primaire & - & 5,8 & 37,3 & 56,8 & - & 23,6 & 36,3 & 40,0 & - & 10,9 & 29,6 & 59,5 \\
\hline $\begin{array}{l}\text { Secondaire et }+ \\
\text { TV }\end{array}$ & - & - & 7,8 & 92,2 & - & 0,5 & 9,1 & 90,3 & - & - & 9,2 & 90,8 \\
\hline Pas du tout & 36,6 & 30,5 & 22,2 & 10,7 & 47,5 & 28,4 & 17,0 & 7,0 & 47,8 & 34,6 & 13,0 & 4,7 \\
\hline Oui/Moins d'une fois par semaine & - & - & 31,8 & 68,2 & 9,1 & 46,4 & 29,6 & 14,9 & - & 32,9 & 41,5 & 25,5 \\
\hline Oui/Au moins une fois par semaine & ND & ND & ND & ND & - & 25,2 & 24,1 & 50,7 & - & 0,8 & 36,5 & 62,7 \\
\hline \multicolumn{13}{|l|}{ Radio } \\
\hline Pas du tout & 63,9 & 10,2 & 19,0 & 6,9 & $5 I, 3$ & 24,3 & 16,4 & 7,9 & 73,5 & 7,8 & 13,9 & 4,9 \\
\hline Oui/Moins d'une fois par semaine & - & 32,6 & 28,8 & 38,6 & $4 I, 6$ & 27,4 & 18,3 & 12,7 & 10,2 & 43,6 & 24,1 & 22,1 \\
\hline $\begin{array}{l}\text { Oui/Au moins une fois par semaine } \\
\text { Journaux }\end{array}$ & ND & ND & ND & ND & 14,6 & 31,0 & 23,1 & 31,3 & - & 26,6 & 33,0 & 40,4 \\
\hline Pas du tout & 28,6 & 23,9 & 25,6 & 21,9 & 25,6 & 30,3 & 21,9 & 22,2 & 26,9 & 26,1 & 26,3 & 20,6 \\
\hline Oui/Moins d'une fois par semaine & - & - & - & 100,0 & - & - & - & 100,0 & - & - & 1,2 & 98,8 \\
\hline $\begin{array}{l}\text { Oui/Au moins une fois par semaine } \\
\text { Occupation femme }\end{array}$ & ND & ND & ND & ND & - & - & - & 100,0 & - & - & - & 100,0 \\
\hline Inactive & 31,1 & 23,1 & 21,7 & 24,1 & 19,4 & 24,9 & 24,1 & 31,6 & 25,0 & 26,2 & 25,2 & 23,6 \\
\hline Cadre/employée & - & - & - & 100,0 & - & - & 5,7 & 94,3 & - & - & - & 100,0 \\
\hline Commerçante & - & 17,8 & 39,4 & 42,8 & - & 22,7 & 29,5 & 47,8 & 10,7 & 16,0 & 31,3 & 41,9 \\
\hline Agricultrice & 41,9 & 29,8 & 22,0 & 6,3 & 45,6 & 36,6 & $14, \mid$ & 3,6 & 48,2 & 32,0 & 15,9 & 3,9 \\
\hline Services & - & 8,7 & 18,1 & 73,2 & 5,0 & 19,8 & $4 I, 6$ & 33,7 & ND & ND & ND & ND \\
\hline Ouvrière & 46,6 & 21,6 & 14,1 & 17,7 & - & 29,9 & 24,8 & 45,3 & ND & ND & ND & ND \\
\hline
\end{tabular}

Notes : ND=variable ou modalité non disponible ; $T$ faible $=$ très faible. 
African Population Studies Vol.33, No. I, Feb. 2019

Annexes $\mathrm{n}^{\circ} 2$ : Résultats des analyses bivariées

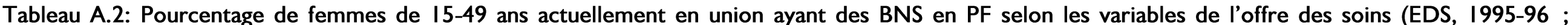
2006 et 2012-13)

\begin{tabular}{|c|c|c|c|c|c|c|c|c|c|c|c|c|}
\hline \multirow[t]{2}{*}{ Variables de l'offre des soins } & \multicolumn{4}{|c|}{ EDS 1995-96 } & \multicolumn{4}{|c|}{ EDS 2006} & \multicolumn{4}{|c|}{ EDS $2012-13$} \\
\hline & Espacer & Limiter & Total & $\mathrm{N}$ & Espacer & Limiter & Total & $N$ & Espacer & Limiter & Total & $\mathrm{N}$ \\
\hline Reçu les visites des ASC & ND & ND & ND & ND & Ns & $* *$ & ns & & ns & $* *$ & $* *$ & \\
\hline Oui & & & & & 25,9 & 9,0 & 34,9 & 1452 & 18,6 & 9,0 & 27,6 & 1030 \\
\hline Non & & & & & 26,6 & 7,6 & 34,2 & 11236 & 18,5 & 7,2 & 25,7 & 7769 \\
\hline $\begin{array}{l}\text { Prestataire lui a parlé de PF } \\
\text { dans le centre de santé lors } \\
\text { de sa dernière consultation } \\
\text { Oui }\end{array}$ & $* *$ & ns & $* *$ & & ** & $* *$ & $* *$ & & *** & ns & $* *$ & \\
\hline Non & 22,9 & 5,0 & 27,9 & 1854 & 26,4 & 6,6 & 33,0 & 1220 & 16,6 & 6,4 & 23,0 & 1067 \\
\hline N'a pas fréquenté le centre & 23,2 & 5,8 & 29,0 & 552 & 29,0 & 6,8 & 35,8 & 2473 & 21,0 & 7,2 & 28,2 & 1204 \\
\hline de santé & 18,7 & 5,9 & 24,6 & 5676 & 25,5 & 8,2 & 33,7 & 8969 & 18,3 & 7,6 & 25,9 & 6528 \\
\hline Ensemble & 20,0 & 5,7 & 25,7 & 8082 & 26,4 & 7,8 & 34,2 & 12688 & 18,5 & 7,4 & 25,9 & 8799 \\
\hline
\end{tabular}

Notes : ** $p \leq 0,05$ lorsqu'on compare au moins deux modalités de la variable ; ns =non significatif ; ND=variable non disponible.

Tableau A.3: Pourcentage de femmes de 15-49 ans actuellement en union ayant des BNS en PF selon les variables opérationnelles du milieu socioculturel traditionnel (EDS, 1995-96; 2006 et 2012-13)

\begin{tabular}{|c|c|c|c|c|c|c|c|c|c|c|c|c|}
\hline \multirow{2}{*}{$\begin{array}{l}\text { Variables opérationnelles du milieu } \\
\text { socioculturel traditionnel } \\
\text { Ethnie }\end{array}$} & \multicolumn{4}{|c|}{ EDS 1995-96 } & \multicolumn{4}{|c|}{ EDS 2006} & \multicolumn{4}{|c|}{ EDS 2012-13 } \\
\hline & $\begin{array}{l}\text { Espacer } \\
* *\end{array}$ & $\begin{array}{l}\text { Limiter } \\
* *\end{array}$ & $\begin{array}{l}\text { Total } \\
* *\end{array}$ & $\mathrm{~N}$ & $\begin{array}{l}\text { Espacer } \\
* *\end{array}$ & $\begin{array}{l}\text { Limiter } \\
* *\end{array}$ & $\begin{array}{l}\text { Total } \\
* *\end{array}$ & $\mathrm{~N}$ & $\begin{array}{l}\text { Espacer } \\
\text { ns }\end{array}$ & $\begin{array}{l}\text { Limiter } \\
* *\end{array}$ & $\begin{array}{l}\text { Total } \\
* *\end{array}$ & $N$ \\
\hline Mandingue & 21,2 & 6,0 & 27,2 & 4044 & 26,7 & 8,6 & 35,3 & 7264 & 18,4 & 7,3 & 25,7 & 4757 \\
\hline Soudanienne & 18,2 & 3,9 & 22,1 & 1038 & 23,4 & 8,0 & 31,4 & 1126 & 18,9 & 7,1 & 26,0 & 1050 \\
\hline Voltaïque & 18,4 & 6,4 & 24,8 & 749 & 24,6 & 7,8 & 32,4 & 1281 & 19,2 & 7,0 & 26,2 & 884 \\
\hline Nomade & 19,8 & 5,7 & 25,5 & 1394 & 26,5 & 5,9 & 32,4 & 2412 & 18,1 & 7,3 & 25,4 & 1524 \\
\hline Autres & 18,4 & 5,6 & 24,0 & 874 & 30,9 & 5,3 & 36,2 & 739 & 18,8 & 10,4 & 29,2 & 584 \\
\hline Religion & $* *$ & ** & ** & & $* *$ & *** & ** & & *** & *** & $* *$ & \\
\hline Musulmane & 20,2 & 5,9 & 26,1 & 7323 & 26,4 & 7,5 & 33,9 & 11654 & 18,7 & 7,3 & 26,0 & 8165 \\
\hline Chrétienne & 19,7 & 5,6 & 25,3 & 233 & 26,7 & 9,9 & 36,6 & 344 & 12,3 & 8,4 & 20,7 & 356 \\
\hline Animiste & 13,8 & 3,1 & 16,9 & 420 & 20,8 & 13,9 & 34,7 & 259 & 19,3 & 13,3 & 32,6 & 82 \\
\hline Autres & 30,5 & 4,6 & 35,1 & $13 \mid$ & 28,5 & 8,9 & 37,4 & 495 & 19,2 & 5,6 & 24,8 & 196 \\
\hline Ensemble & 20,0 & 5,7 & 25,7 & 8082 & 26,4 & 7,8 & 34,2 & 12688 & 18,5 & 7,4 & 25,9 & 8799 \\
\hline
\end{tabular}

Notes : ** $\mathrm{p} \leq 0,05$ lorsqu'on compare au moins deux modalités de la variable; $n s=$ non significatif. 
Tableau A.4: Pourcentage de femmes de 15-49 ans actuellement en union ayant des BNS en PF selon le degré de modernité culturelle (EDS, 1995-96 ; 2006 et 2012-13)

\begin{tabular}{|c|c|c|c|c|c|c|c|c|c|c|c|c|}
\hline \multirow[t]{2}{*}{ Degré modernité culturelle } & \multicolumn{4}{|c|}{ EDS $1995-96$} & \multicolumn{4}{|c|}{ EDS 2006} & \multicolumn{4}{|c|}{ EDS 2012-13 } \\
\hline & $\begin{array}{l}\text { Espacer } \\
* *\end{array}$ & $\begin{array}{l}\text { Limiter } \\
* *\end{array}$ & $\begin{array}{l}\text { Total } \\
\text { *** }\end{array}$ & $\mathrm{N}$ & $\begin{array}{l}\text { Espacer } \\
* * *\end{array}$ & $\begin{array}{l}\text { Limiter } \\
* *\end{array}$ & $\begin{array}{l}\text { Total } \\
* *\end{array}$ & $\mathrm{~N}$ & $\begin{array}{l}\text { Espacer } \\
* *\end{array}$ & $\begin{array}{l}\text { Limiter } \\
\text { *** }\end{array}$ & $\begin{array}{l}\text { Total } \\
* *\end{array}$ & $\mathrm{~N}$ \\
\hline Très faible & 16,9 & 5,0 & 21,9 & 2116 & 23,3 & 8,7 & 32,0 & 2991 & $16, \mid$ & 8,1 & 24,2 & 1908 \\
\hline Faible & 20,1 & 5,6 & 25,7 & 1763 & 25,1 & 8,7 & 33,8 & 3535 & 18,3 & 8,8 & 27,1 & $|85|$ \\
\hline Moyen & 21,2 & 6,4 & 27,6 & 1892 & 28,1 & 7,6 & 35,7 & 2559 & 20,5 & 7,7 & 28,2 & $|87|$ \\
\hline Elevé & 22,3 & 5,6 & 27,9 & 1923 & 29,5 & 6,6 & 36,1 & 2977 & 19,0 & 5,6 & 24,6 & 1878 \\
\hline Ensemble & 20,0 & 5,7 & 25,7 & 7694 & 26,4 & 7,8 & 34,2 & 12062 & 18,5 & 7,4 & 25,9 & 7508 \\
\hline
\end{tabular}

Notes : Idem tableau A.2.

Tableau A.5: Pourcentage de femmes de I5-49 ans actuellement en union ayant des BNS en PF selon les indicateurs de la modernité économique (EDS, 1995$96 ; 2006$ et $2012-13)$

\begin{tabular}{|c|c|c|c|c|c|c|c|c|c|c|c|c|}
\hline \multirow{2}{*}{$\begin{array}{l}\text { Indicateurs de la modernité } \\
\text { économique }\end{array}$} & \multicolumn{4}{|c|}{ EDS 1995-96 } & \multicolumn{4}{|c|}{ EDS 2006} & \multicolumn{4}{|c|}{ EDS 2012-13 } \\
\hline & Espacer & Limiter & Total & $\mathrm{N}$ & Espacer & Limiter & Total & $N$ & Espacer & Limiter & Total & $\mathrm{N}$ \\
\hline Occupation conjoint & $* *$ & ** & *** & & $* *$ & ** & $* *$ & & ND & ND & ND & ND \\
\hline Inactifs & 22,9 & 2,3 & 25,2 & $|3|$ & - & - & - & - & & & & \\
\hline Cadres/employés & 17,2 & 6,4 & 23,6 & 424 & 20,4 & 7,0 & 27,4 & 500 & & & & \\
\hline Commerçants & 22,8 & 5,6 & 28,4 & 712 & 29,8 & 5,0 & 34,8 & 1805 & & & & \\
\hline Agriculteurs & 19,3 & 5,4 & 24,7 & 5460 & 25,2 & 8,6 & 33,8 & 8165 & & & & \\
\hline Services & 22,7 & 7,4 & 30,1 & 934 & - & - & - & - & & & & \\
\hline Ouvriers & 20,3 & 5,5 & 25,8 & 433 & 28,8 & 8,4 & 37,2 & 1387 & & & & \\
\hline Autres & - & - & - & - & 30,0 & 7,1 & 37,1 & 283 & & & & \\
\hline Niveau de vie & ND & ND & ND & ND & *** & *** & $* *$ & & **⿻丷木 & *** & *** & \\
\hline Très pauvre & & & & & 27,3 & 7,7 & 35,0 & 2811 & 17,9 & 7,1 & 25,0 & 1767 \\
\hline Pauvre & & & & & 25,9 & 9,1 & 35,0 & 2663 & 16,8 & 8,6 & 25,4 & $|77|$ \\
\hline Moyen & & & & & 24,7 & 9,2 & 33,9 & 2618 & 19,8 & 8,4 & 28,2 & 1774 \\
\hline Riche & & & & & 25,3 & 6,7 & 32,0 & 2513 & 20,9 & 6,6 & 27,5 & 1779 \\
\hline Très riche & & & & & 28,8 & 5,9 & 34,7 & 2200 & 16,9 & 6,2 & 23,1 & 1708 \\
\hline Ensemble & 20,0 & 5,7 & 25,7 & 8094 & 26,4 & 7,8 & 34,2 & 12729 & 18,5 & 7,4 & 25,9 & 8799 \\
\hline
\end{tabular}

Notes: ** $\mathrm{p} \leq 0,05$ lorsqu'on compare au moins deux modalités de la variable ; ND=variable non disponible. 
African Population Studies Vol.33, No.I, Feb. 2019

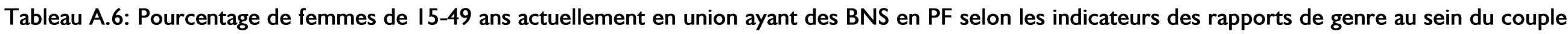
(EDS, I995-96 et 2006)

\begin{tabular}{|c|c|c|c|c|c|c|c|c|}
\hline \multirow[t]{2}{*}{ Indicateurs des rapports de genre } & \multicolumn{4}{|c|}{ EDS 1995-96 } & \multicolumn{4}{|c|}{ EDS 2006} \\
\hline & Espacer & Limiter & Total & $\mathrm{N}$ & Espacer & Limiter & Total & $\mathrm{N}$ \\
\hline Attitudes du couple vis-à-vis de la PF & *** & *** & *** & & 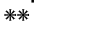 & *** & 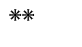 & \\
\hline \multicolumn{9}{|l|}{ Approuve } \\
\hline Désapprouve & 23,6 & 7,9 & 31,5 & 1968 & 27,4 & 7,6 & 35,0 & 3455 \\
\hline Femme seule & 15,9 & 2,7 & 18,6 & 1055 & 18,1 & 5,0 & 23,1 & 2349 \\
\hline \multirow[t]{2}{*}{ Conjoint seul } & 25,7 & 6,9 & 32,6 & 1060 & 28,1 & 11,1 & 39,2 & 2111 \\
\hline & 16,7 & 7,1 & 23,8 & 98 & 15,6 & 14,3 & 29,9 & 147 \\
\hline \multicolumn{9}{|l|}{ Pas du tout } \\
\hline Une ou deux fois & 19,0 & 5,0 & 24,0 & 5289 & 26,2 & 7,4 & 33,6 & 7961 \\
\hline \multirow[t]{3}{*}{ Souvent } & 20,7 & 6,0 & 26,7 & 1508 & 28,6 & 8,1 & 34,7 & 2443 \\
\hline & 23,3 & 8,0 & 31,3 & 1300 & 24,3 & 8,7 & 33,0 & 2303 \\
\hline & 20,0 & 5,7 & 25,7 & 8097 & 26,4 & 7,8 & 34,2 & 12707 \\
\hline
\end{tabular}

Notes: Idem tableau A.2.

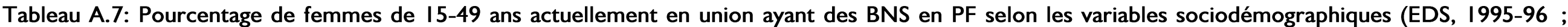
2006 et 2012-13)

\begin{tabular}{|c|c|c|c|c|c|c|c|c|c|c|c|c|}
\hline \multirow{2}{*}{ Variables sociodémographiques } & \multicolumn{4}{|c|}{ EDS 1995-96 } & \multicolumn{4}{|l|}{ EDS 2006} & \multicolumn{4}{|c|}{ EDS 2012-13 } \\
\hline & $\begin{array}{l}\text { Espacer } \\
\text { *** }\end{array}$ & $\begin{array}{l}\text { Limiter } \\
\text { **⿻丷木 }\end{array}$ & $\begin{array}{l}\text { Total } \\
\text { **⿻丷木 }\end{array}$ & $\mathrm{N}$ & $\begin{array}{l}\text { Espacer } \\
\text { *⿻丷木 }\end{array}$ & $\begin{array}{l}\text { Limiter } \\
\text { **⿻丷木 }\end{array}$ & $\begin{array}{l}\text { Total } \\
\text { **⿻丷木 }\end{array}$ & $\mathrm{N}$ & $\begin{array}{l}\text { Espacer } \\
\text { *** }\end{array}$ & $\begin{array}{l}\text { Limiter } \\
\text { **⿻丷木 }\end{array}$ & $\begin{array}{l}\text { Total } \\
\text { **⿻丷木 }\end{array}$ & $\mathrm{N}$ \\
\hline Moins de 15 & 21,0 & 7,5 & 28,5 & 1868 & 24,7 & 9,2 & 33,9 & 3358 & 18,0 & 8,1 & 28,1 & 2077 \\
\hline 15 & 20,6 & 5,3 & 25,9 & 2468 & 27,9 & 7,7 & 35,6 & 2573 & 18,8 & 6,7 & 25,5 & 1110 \\
\hline $16-18$ & 19,6 & 5,6 & 25,2 & 2678 & 26,6 & 6,2 & 32,8 & 4501 & 19,4 & 7,4 & 26,8 & 2740 \\
\hline $19-24$ & 18,8 & 3,8 & 22,6 & 957 & 26,6 & 8,8 & 35,4 & 2061 & 18,8 & 6,8 & 25,6 & 2250 \\
\hline $25+$ & 12,4 & 1,6 & 14,0 & 129 & 27,4 & 9,7 & 37,1 & 236 & 14,6 & 8,4 & 23,0 & 622 \\
\hline Proportion enfants décédés (en \%) & *** & *** & *** & & *** & *** & *** & & *** & *** & *** & \\
\hline 0 & 24,5 & 3,2 & 27,7 & 2829 & 27,3 & 4,8 & 32,1 & 5789 & 20,1 & 6,6 & 26,7 & 5958 \\
\hline $7-24$ & 21,4 & 15,6 & 37,0 & 1122 & 22,4 & 16,1 & 38,5 & 1798 & 15,9 & 14,8 & 30,7 & 779 \\
\hline $25-39$ & 19,2 & 7,9 & 27,1 & 1475 & 25,8 & 11,0 & 36,8 & 2546 & 18,6 & 13,1 & 31,7 & 796 \\
\hline $40-59$ & 17,6 & 5,0 & 22,6 & 1300 & 31,5 & 6,2 & 37,7 & 1849 & 16,6 & 7,0 & 23,6 & 471 \\
\hline $60+$ & 9,9 & 1,8 & 11,7 & 735 & 18,1 & 3,7 & 21,8 & 747 & 7,9 & 2,9 & 10,5 & 240 \\
\hline Nombre enfants survivants & *** & *** & *** & & *** & *** & *** & & *** & *** & *** & \\
\hline 0 & 14,1 & 0,0 & $|4|$, & 891 & 10,1 & 0,9 & 11,0 & 216 & 11,0 & 0,6 & 11,6 & 710 \\
\hline I & 22,2 & 0,2 & 22,4 & 1323 & 29,4 & 0,8 & 30,2 & 1914 & 20,9 & 1,0 & 21,9 & 1369 \\
\hline 2 & 22,7 & $\mathrm{I}, 0$ & 23,7 & $126 \mid$ & 29,8 & $\mathrm{I}, 7$ & 31,5 & 2439 & 18,6 & 1,5 & 20,1 & 1426 \\
\hline 3 & 21,8 & 2,1 & 23,9 & 1178 & 30,9 & 2,6 & 33,5 & 2403 & 21,7 & 4,2 & 25,9 & 1416 \\
\hline 4 & 22,1 & 4,2 & 26,3 & 1092 & 28,2 & 5,5 & 33,7 & 1874 & 21,6 & 7,0 & 28,6 & 1352 \\
\hline 5 & 21,3 & 8,9 & 30,2 & 870 & 20,6 & 11,4 & 32,0 & 1428 & 16,0 & 12,6 & 28,6 & 1041 \\
\hline $6+$ & 15,5 & 20,0 & 35,5 & 1492 & 19,5 & 24,7 & 44,2 & 2455 & 15,6 & 22,0 & 37,6 & 1485 \\
\hline Ensemble & 20,0 & 5,7 & 25,7 & 8094 & 26,4 & 7,8 & 34,2 & 12729 & 18,5 & 7,4 & 25,9 & 8799 \\
\hline
\end{tabular}

Notes: ** $\mathrm{p} \leq 0,05$ lorsqu'on compare au moins deux modalités de la variable. 
Tableau A.8: Application de la méthode de décomposition simple à l'analyse du changement de la proportion des femmes ayant des BNS en PF pour espacer les naissances de 1995 à 2006.

\begin{tabular}{|c|c|c|c|c|c|c|}
\hline \multirow[t]{2}{*}{ Variables + modalités } & \multicolumn{2}{|c|}{ Année 1995-96 } & \multicolumn{2}{|c|}{ Année 2006} & \multicolumn{2}{|c|}{ \% changement lié à } \\
\hline & $P$ & $\%$ & $P$ & $\%$ & Composition & Comportement \\
\hline \multicolumn{7}{|c|}{ Niveau d'instruction de la femme } \\
\hline Primaire & 20,1 & 85,2 & 26,4 & 85,7 & 0,109 & 5,385 \\
\hline Secondaire ou supérieur & 21,0 & 10,3 & 25,9 & 10,1 & $-0,035$ & 0,499 \\
\hline \multirow[t]{2}{*}{ Total } & 15,1 & 4,5 & 27,4 & 4,2 & $-0,068$ & 0,534 \\
\hline & 20,0 & 100,0 & 26,4 & 100,0 & $\begin{array}{l}0,006 \\
0,09 \%\end{array}$ & $\begin{array}{l}6,418 \\
99,91 \%\end{array}$ \\
\hline \multicolumn{7}{|c|}{ Niveau d'instruction du conjoint } \\
\hline Primaire & 19,7 & 83,8 & 26,6 & 80,8 & $-0,694$ & 5,385 \\
\hline Secondaire ou supérieur & 22,5 & 8,0 & 25,9 & 11,0 & 0,740 & 0,499 \\
\hline \multirow[t]{3}{*}{ Total } & 20,1 & 8,2 & 24,2 & 8,2 & $-0,016$ & 0,534 \\
\hline & 20,0 & 100,0 & 26,4 & 100,0 & 0,030 & 6,418 \\
\hline & & & & & $0,47 \%$ & $99,53 \%$ \\
\hline \multicolumn{7}{|c|}{ Nombre d'enfants survivants } \\
\hline I & 14,1 & 11,0 & 10,1 & $\mathrm{I}, 7$ & $-1,125$ & $-0,254$ \\
\hline 2 & 22,2 & 16,3 & 29,4 & 15,0 & $-0,335$ & $\mathrm{I}, \mathrm{I} 27$ \\
\hline 3 & 22,7 & 15,6 & 29,8 & 19,2 & 0,945 & $\mathrm{I}, 235$ \\
\hline 4 & 21,8 & 14,5 & 30,9 & 18,9 & 1,159 & 1,520 \\
\hline 5 & 22,1 & 13,5 & 28,2 & 14,7 & 0,302 & 0,860 \\
\hline 6 & 21,3 & 10,7 & 20,6 & 11,2 & 0,105 & $-0,077$ \\
\hline \multirow[t]{3}{*}{ Total } & 15,5 & 18,4 & 19,5 & 19,3 & 0,158 & 0,754 \\
\hline & 20,0 & 100,0 & 26,4 & 100,0 & 1,209 & 5,165 \\
\hline & & & & & $18,97 \%$ & $81,03 \%$ \\
\hline
\end{tabular}

La lecture de ces résultats se fait comme suit : l'augmentation en 1995-2006 de la proportion des femmes ayant des BNS en PF pour espacer les naissances (de 20 $\%$ à $26,4 \%$ ) est plus liée à l'amélioration de leurs préférences en matière de fécondité qu'au changement de la structure de la population au niveau des variables considérées. En effet, dans le cas du niveau d'instruction de la femme, presque la totalité de l'augmentation observée au cours de cette période provient de l'effet de comportement $(99,91 \%)$. En revanche, concernant le nombre d'enfants survivants, l'effet de composition (18,97\%) n'est pas négligeable, bien qu'il reste plus faible que celui de comportement $(81,03 \%)$. Les femmes sans niveau d'instruction ont le plus contribué à l'augmentation de la proportion d'individus ayant des BNS en PF pour espacer les naissances. Concernant le nombre d'enfants survivants, les femmes ayant peu d'enfants et celles qui en ont beaucoup ont toutes contribué à cette augmentation au cours de la période considérée. 
Tableau A.9: Application de la méthode de décomposition simple à l'analyse du changement de la proportion des femmes ayant des BNS en PF pour limiter les naissances de 1995 à 2006.

\begin{tabular}{|c|c|c|c|c|c|c|}
\hline \multirow[t]{2}{*}{ Variables + modalités } & \multicolumn{2}{|c|}{ Année 1995-96 } & \multicolumn{2}{|c|}{ Année 2006} & \multicolumn{2}{|c|}{ \% changement lié à } \\
\hline & $P$ & $\%$ & $P$ & $\%$ & Composition & Comportement \\
\hline \multicolumn{7}{|c|}{ Niveau d'instruction de la femme } \\
\hline Sans niveau & 5,7 & 85,2 & 8,0 & 85,7 & 0,034 & 1,965 \\
\hline Primaire & 5,9 & 10,3 & 7,5 & 10,1 & $-0,013$ & 0,163 \\
\hline Secondaire ou supérieur & 5,5 & 4,5 & 4,7 & 4,2 & $-0,015$ & $-0,044$ \\
\hline \multirow[t]{2}{*}{ Total } & 5,7 & 100,0 & 7,8 & 100,0 & 0,006 & 2,085 \\
\hline & & & & & $0,29 \%$ & $99,71 \%$ \\
\hline \multicolumn{7}{|c|}{ Niveau d'instruction du conjoint } \\
\hline Sans niveau & 5,7 & 83,8 & 8,1 & 80,8 & $-0,207$ & I,975 \\
\hline Primaire & 5,6 & 8,0 & 7,6 & 11,0 & 0,198 & 0,190 \\
\hline Secondaire ou supérieur & 5,3 & 8,2 & 5,5 & 8,2 & 0 & 0,016 \\
\hline \multirow[t]{2}{*}{ Total } & 5,7 & 100,0 & 7,8 & 100,0 & $-0,009$ & 2,182 \\
\hline & & & & & $-0,41 \%$ & $100,4 \mid \%$ \\
\hline \multicolumn{7}{|c|}{ Nombre d'enfants survivants } \\
\hline 0 & 0,0 & 11,0 & 0,9 & I,7 & $-0,042$ & 0,057 \\
\hline I & 0,2 & 16,3 & 0,8 & 15,0 & $-0,007$ & 0,094 \\
\hline 2 & 1,0 & 15,6 & $\mathrm{I}, 7$ & 19,2 & 0,049 & 0,122 \\
\hline 3 & 2,1 & 14,5 & 2,6 & 18,9 & 0,103 & 0,084 \\
\hline 4 & 4,2 & 13,5 & 5,5 & 14,7 & 0,058 & 0,183 \\
\hline 5 & 8,9 & 10,7 & 11,4 & 11,2 & 0,058 & 0,274 \\
\hline 6 & 20,0 & 18,4 & 24,7 & 19,3 & 0,201 & 0,886 \\
\hline \multirow[t]{2}{*}{ Total } & 5,7 & 100,0 & 7,8 & 100,0 & $0,4 \mid 4$ & 1,699 \\
\hline & & & & & $19,6 \%$ & $80,4 \%$ \\
\hline
\end{tabular}

Les sources de l'augmentation de la proportion des femmes ayant des BNS pour limiter les naissances (de $5,7 \%$ à $7,8 \%$ ) sont similaires à celles mises en évidence dans le cas de l'espacement des naissances. Mais, dans le cas du nombre d'enfants survivants, ce sont les femmes qui ont au moins 4 enfants en vie qui ont le plus contribué à l'augmentation de cette proportion. Ces résultats confortent l'idée selon laquelle les préférences des femmes dans le domaine de la fécondité se sont améliorées au cours de la période 1995-2006 dans le pays étudié mais ceci s'est accompagné de l'augmentation de la fréquence de celles ayant des BNS pour espacer ou limiter les naissances, à cause d'une offre en PF quantitativement et qualitativement insuffisante au cours de cette période. 
African Population Studies Vol.33, No. I, Feb. 2019

Tableau A. I0: Application de la méthode de décomposition simple à l'analyse du changement de la proportion des femmes ayant des BNS en PF pour espacer les naissances de 2006 à 2013.

\begin{tabular}{|c|c|c|c|c|c|c|}
\hline \multirow[t]{2}{*}{ Variables + modalités } & \multicolumn{2}{|c|}{ Année 2006} & \multicolumn{2}{|c|}{ Année 2012-13 } & \multicolumn{2}{|c|}{ \% changement lié à } \\
\hline & $\mathrm{P}$ & $\%$ & $P$ & $\%$ & Composition & Comportement \\
\hline \multicolumn{7}{|c|}{ Niveau d'instruction de la femme } \\
\hline Sans niveau & 26,4 & 85,7 & 18,2 & 82,1 & $-0,803$ & $-6,880$ \\
\hline Primaire & 25,9 & 10,1 & 20,8 & 8,8 & $-0,304$ & $-0,482$ \\
\hline Secondaire ou supérieur & 27,4 & 4,2 & 18,6 & 9,1 & $\mathrm{I}, \mathrm{I} 27$ & $-0,585$ \\
\hline \multirow[t]{2}{*}{ Total } & 26,4 & 100,0 & 18,5 & 100,0 & 0,021 & $-7,947$ \\
\hline & & & & & $0,3 \%$ & $100,3 \%$ \\
\hline \multicolumn{7}{|c|}{ Niveau d'instruction du conjoint } \\
\hline Sans niveau & 26,6 & 80,8 & 19,1 & 81,0 & 0,046 & $-6,067$ \\
\hline Primaire & 25,9 & $I I, 0$ & 17,1 & 7,0 & $-0,860$ & $-0,792$ \\
\hline Secondaire ou supérieur & 24,2 & 8,2 & 15,5 & 12,0 & 0,754 & $-0,879$ \\
\hline \multirow[t]{2}{*}{ Total } & 26,4 & 100,0 & 18,5 & 100,0 & $-0,060$ & $-7,738$ \\
\hline & & & & & $0,77 \%$ & $99,23 \%$ \\
\hline \multicolumn{7}{|c|}{ Nombre d'enfants survivants } \\
\hline 0 & 10,1 & $\mathrm{I}, 7$ & $I I, 0$ & 8,1 & 0,675 & 0,044 \\
\hline I & 29,4 & 15,0 & 20,9 & 15,6 & 0,151 & $-I, 301$ \\
\hline 2 & 29,8 & 19,2 & 18,6 & 16,2 & $-0,726$ & $-1,982$ \\
\hline 3 & 30,9 & 18,9 & 21,7 & 16,1 & $-0,736$ & $-1,610$ \\
\hline 4 & 28,2 & 14,7 & 21,6 & 15,3 & 0,149 & $-0,990$ \\
\hline 5 & 20,6 & II,2 & 16,0 & 11,8 & 0,110 & $-0,529$ \\
\hline 6 & 19,5 & 19,3 & 15,6 & 16,9 & $-0,421$ & $-0,706$ \\
\hline \multirow[t]{2}{*}{ Total } & 26,4 & 100,0 & 18,5 & 100,0 & $-0,798$ & $-7,074$ \\
\hline & & & & & $10,14 \%$ & $89,86 \%$ \\
\hline
\end{tabular}


Quelle que soit la variable de différentiation considérée, l'effet de comportement explique davantage la baisse de la proportion des femmes ayant des BNS en PF pour espacer les naissances (de $26,4 \%$ en 2006 à $18,5 \%$ en 2013 ) que l'effet de composition: $100,3 \%$ vs $0,3 \%$ dans le cas du niveau d'instruction de la femme et $89,86 \%$ vs $10,14 \%$ dans celui du nombre d'enfants survivants. Les femmes sans niveau d'instruction ou ayant au plus 4 enfants en vie ont le plus contribué à cette baisse. L'idée selon laquelle l'amélioration de l'offre en PF en 2006-2013 a permis aux femmes de satisfaire en partie leurs besoins en PF pour espacer les naissances serait donc pertinente ici.

En 2006-20 I3,la proportion de femmes ayant des BNS en PF pour limiter les naissances n'a pas significativement changé dans le temps $(7,8 \%$ en 2006 et $7,4 \%$ en 20 । 3 ). 